\title{
A Polygonal Discontinuous Galerkin Formulation for Contact Mechanics in Fluid-Structure Interaction Problems
}

\author{
Stefano Zonca ${ }^{1, *}$, Paola F. Antonietti ${ }^{1}$ and Christian Vergara ${ }^{2}$ \\ ${ }^{1}$ MOX, Dipartimento di Matematica, Politecnico di Milano, Italy. \\ 2 LABS, Dipartimento di Chimica, Materiali e Ingegneria Chimica "Giulio Natta", \\ Politecnico di Milano, Italy.
}

Received 6 May 2020; Accepted (in revised version) 2 December 2020

\begin{abstract}
In this work, we propose a formulation based on the Polygonal Discontinuous Galerkin (PolyDG) method for contact mechanics that arises in fluid-structure interaction problems. In particular, we introduce a consistent penalization approach to treat the frictionless contact between immersed structures that undergo large displacements. The key feature of the method is that the contact condition can be naturally embedded in the PolyDG formulation, allowing to easily support polygonal/polyhedral meshes. The proposed approach introduced a fixed background mesh for the fluid problem overlapped by the structure grid that is able to move independently of the fluid one. To assess the validity of the proposed approach, we report the results of several numerical experiments obtained in the case of contact between flexible structures immersed in a fluid.
\end{abstract}

AMS subject classifications: $65 \mathrm{M} 60,75 \mathrm{~F} 10,74 \mathrm{M} 15$

Key words: Polygonal Discontinuous Galerkin method, fluid-structure interaction, contact mechanics.

\section{Introduction}

The numerical modeling of the contact process in mechanical problems requires to introduce inequalities in the mathematical formulation, see e.g. [60, 85]. In particular, the contact could be modeled by means of two inequalities, one prescribing a kinematic nonpenetration condition on the displacements, the other one prescribing a dynamic condition on the tractions; an additional equality ensuring the compatibility between these two

*Corresponding author. Email address: stefano.zonca@polimi.it (S. Zonca), paola.antonietti@polimi.it (P. F. Antonietti), christian.vergara@polimi.it (C. Vergara) 
conditions is added to the model, see e.g. $[60,85]$. Moreover, depending on the application of interest, a friction law may be prescribed to model the tangential behaviour of the contact tractions [60]. From the numerical viewpoint, these conditions can be imposed via different approaches. In [38], a penalty method is employed to impose the kinematic nonpenetration condition. In $[35-37,39,40,69]$ and $[25-27,76]$, the authors used a Nitsche-DG and augmented Lagrangian formulations, respectively, that guarantee the consistency of the numerical formulation. In $[41,44,58,59,61,75,83,84]$, a Lagrange multipliers technique is presented for prescribing both the kinematic and the dynamic non-penetration conditions.

Another important process involving many engineering applications is the interaction between a solid body and a fluid, see e.g. [19, 23, 46-48, 52, 53, 67, 72, 86, 87]. The inclusion of the contact model in a fluid-structure interaction (FSI) framework features additional challenges from the mathematical and numerical point of view, due to the need of modeling fluid slip at the fluid-structure interface, a condition that allows contact to take place. There are several applications that require the numerical approximation of FSI problems involving contact mechanics. For example, in the context of the modeling of the cardiovascular system, we mention the dynamics of the heart valves, see e.g. $[12,21,54,65,74,80,81]$.

The physical process that takes place during the contact between two bodies immersed in a fluid has not been completely understood yet, though several recent works addressed the physical behaviour through experiments, see e.g. [18,62,71]. For this reason, the derivation of a suitable mathematical model that describes the contact in FSI with proper coupling conditions is not fully understood. From the theoretical viewpoint, several works show that the contact may happen only under specific conditions on the fluid and structure, or on the topology, see e.g. $[13,34,50,51,57]$. In particular, an important result $[13,34]$ shows that in the case of an incompressible fluid, for a smooth structure geometry, the standard no-slip kinematic coupling condition at the fluid-structure interface does not allow the contact between approaching bodies, since a thin fluid layer remains trapped between them. Thus, it is required to consider a slip condition to allow the contact.

For the numerical treatment of the contact, several approaches have been proposed in the literature. In [77], the authors proposed a fully-Eulerian approach to discretize the FSI problem by using no-slip conditions at the fluid-structure interface. This choice avoids the penetration between the structures, though it never allows their actual contact. In [49], the fully-Eulerian approach has been extended to the contact case with a penalization approach to prevent the penetration of the structures. In [78], the authors proposed a penalty approach to treat the contact problem in the framework of the SpaceTime Finite Element method developed in [79]; in [20], a Fictitious Domain approach for the FSI problem with immersed thin structures is proposed and a penalty approach is employed to incorporate the non-penetration condition into the formulation. Lagrange multiplier approaches have been used as well to handle the contact conditions in FSI, see e.g. $[12,45,68,80]$. 
Recently, a wide range of literature has been focused of the development of consistent penalization methods, such as the Discontinuous Galerkin (DG) scheme. In [24], a fully-Eulerian approach is used to formulate the discrete FSI problem and the DG approach is employed at the interface to prescribe a frictionless contact condition with both no-slip and slip couplings at the fluid-structure interface. The authors prove a stability result and show some two-dimensional numerical examples obtained with conforming meshes. In [2], the Cut Finite Element Method (Cut-FEM) is employed to discretize the FSI problem and frictionless contact conditions are included via a consistent penalty method. In addition, it is proposed a transition from no-slip to slip coupling condition close to the contact limit, based on the general Navier condition, see e.g. $[62,71]$. The authors present two-dimensional numerical examples with unfitted meshes in the case of contact between flexible structures immersed in a fluid. Finally, in [1], the authors extended the previous contact model by coupling it with a poroelastic model to represent the surface roughness in a layer surrounding the structures.

Despite the great advances made, owing to the above-mentioned works, for the FSI contact numerical modeling, some points need in our opinion to be still developed and improved: i) the standard Finite Element method and its extensions, such as the eXtended Finite Element Method (XFEM), use the Lagrangian basis functions that do not allow to easily implement a high-order space discretization. This could be of great interest in some applications, such as to reduce dissipation and dispersion in numerical schemes for elastodynamics problems; ii) since the XFEM uses standard mesh element shapes, such as triangles and quadrilaterals, from the implementation point of view in general it requires a specific treatment of the fluid polygonal elements resulting from the intersection with the structure and of the corresponding degrees of freedom (for example their doubling).

The aim of this work is to design a method that could give some concrete answers to the previous open issues. In particular, we propose a DG formulation for the contact problem in presence of the interaction with a surrounding fluid, valid for meshes with arbitrary polygonal elements. This allows us to develop a consistent, high-order accurate and geometrically flexible numerical method. In particular, the idea is to use discontinuous Finite Elements modal basis functions which are built directly on the polygons generated by the intersections between fluid and structure. This allows us to i) easily implement any order of discretization and ii) manage the degrees of freedom of the cut elements directly on the physical polygons, simplifying their treatment. In the class of approaches based on polygonal elements, we mention [15] for an example of a FSI problem discretized via the Virtual Element method.

Here, we consider the Discontinuous Galerkin method on polygonal grids (PolyDG) $[6,8-10,14,28-32,64,73,82]$ and, in particular, the FSI formulation presented in [11]. Starting from this work, we propose a new consistent contact formulation for the FSI contact problem, where the contact conditions are written in the framework of the PolyDG approach. This allows us to naturally incorporate these conditions in the formulation. As a consequence, we are able to present a scheme that naturally features high-order space 
accuracy for the contact FSI problem and can easily handle non-conforming arbitrarily shaped fluid and structure meshes. This provides us with a gain in geometric flexibility. Notice also that the proposed formulation is valid both for the two and three-dimensional cases.

The paper is organized as follows. In Section 2, we introduce the FSI problem and the contact model; in Section 3, we present the PolyDG contact FSI formulation; finally, in Section 4, we show several 2D numerical experiments to assess the performance of the proposed formulation.

\section{Fluid-structure interaction and contact model}

In this section, we introduce the governing equations for the FSI problem and for the contact model.

Referring to Fig. 1 , for any time $t \in[0, T]$, being $T>0$ the final observation time, we consider a fluid domain $\Omega_{f}(t) \subset \mathbb{R}^{2}$ and a structure domain $\Omega_{s}(t) \subset \mathbb{R}^{2}$ such that $\Omega=$ $\Omega_{f}(t) \cup \Omega_{s}(t), \Omega_{f}(t) \cap \Omega_{s}(t)=\varnothing$. We assume that both the fluid and structure domains are polygonal.

We also assume that the structure domain is completely immersed in the fluid one, so that $\partial \Omega_{s}(t)$ represents the fluid-structure interface. We denote by $\boldsymbol{n}_{i}(t)$ and $\boldsymbol{\tau}_{i}(t)$ the outward unit normal and the unit tangential vectors to $\partial \Omega_{i}(t)$, respectively, for $i=f$,s. We also set $\boldsymbol{n}=\boldsymbol{n}_{f}=-\boldsymbol{n}_{s}$ and $\boldsymbol{\tau}=\boldsymbol{\tau}_{f}=-\boldsymbol{\tau}_{s}$ on $\partial \Omega_{s}(t)$. To ease the notation, when it is not necessary, we drop the dependence on time. For the sake of exposition, we also assume that the bottom boundary of $\Omega_{f}(t)$ is a straight line defined as $\Gamma_{w}=\{(x, y): y=0\}$ that represents a rigid wall with outward vector normal $\boldsymbol{n}_{w}$ and unit tangential vector $\tau_{w}$. We indicate with $\Gamma_{f}$ the fixed external boundary of the fluid domain. In fact, we are considering the case of one immersed structure entering in contact with a rigid wall. The case of two flexible immersed structures will be numerically addressed in Section 3.3.

Our mathematical model is based on the following two assumptions, which accordingly introduce a splitting of $\partial \Omega_{s}$ in three subregions $\Gamma_{C}, \Sigma_{\text {slip }}, \Sigma_{\text {no-slip }}$, where different coupling conditions will be applied:

i) A thin layer of fluid is assumed to be always present between structures, also at the contact region. The latter is denoted by $\Gamma_{C}$, see Fig. 1 . Thus, the bodies may directly exchange forces even though their geometries do not get in touch. In particular, we assume that the contact happens whenever the distance between the two bodies is lower than $\varepsilon>0$, for a suitable $\varepsilon$. This choice is introduced to simplify the resulting numerical approximation, see also Remark 2.1. Owing to this, the fluid structure interface is still $\partial \Omega_{S}$ also during the contact;

ii) To allow the contact between the bodies, we need to prescribe slip conditions between fluid and structure at the contact region. We extend to $\Sigma_{\text {slip }}$ the region where slip conditions are prescribed, even if no contact occurs here, see Fig. 1 . This is done 


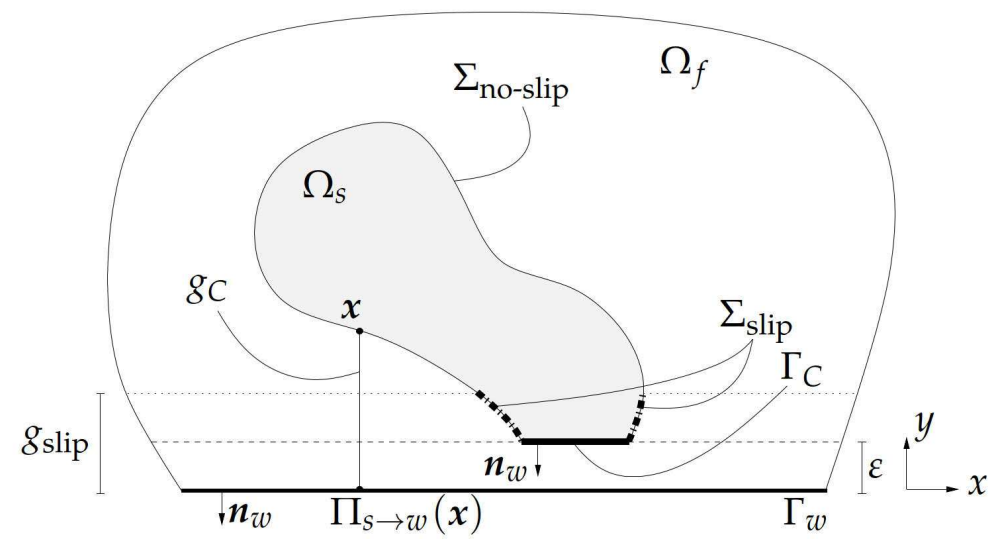

Figure 1: Sketch of the fluid and structure domains $\Omega_{f}$ and $\Omega_{s}$ with the contact conditions. $\Gamma_{w}$ is the rigid

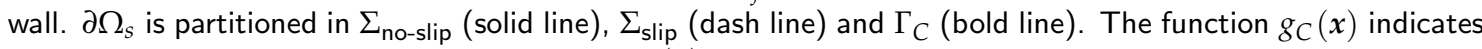
the distance between $x$ and its projection $\Pi_{s \rightarrow w}(\boldsymbol{x})$ on $\Gamma_{w}$. The threshold distance $g_{\text {slip }}$ separates the $\Sigma_{\text {no-slip }}$ and $\Sigma_{\text {slip }}$ regions, while $\varepsilon$ separates the $\Sigma_{\text {slip }}$ and $\Gamma_{C}$ regions.

in order to allow the fluid between the flexible and rigid structures to slip away, so that they could move closer each other.

On the remaining part of the interface, $\Sigma_{\text {no-slip, }}$ standard FSI coupling conditions are prescribed, see Fig. 1.

The structure problem is written in a Lagrangian framework; accordingly, the quantities in the reference configuration are denoted with $\widehat{\imath}$. The fluid and contact problems are instead written in an Eulerian framework.

We consider an incompressible Newtonian fluid with $\boldsymbol{T}_{f}(\boldsymbol{u}, p)=-p \boldsymbol{I}+2 \mu_{f} \boldsymbol{D}(\boldsymbol{u})$, where $\boldsymbol{D}(\boldsymbol{v})=\frac{1}{2}\left(\nabla \boldsymbol{v}+\nabla \boldsymbol{v}^{T}\right), \boldsymbol{u}$ and $p$ are the fluid velocity and pressure and $\mu_{f}$ is the fluid dynamic viscosity. For the structure, we consider a linear elastic material with $T_{s}(d)=$ $\lambda_{s}(\nabla \cdot \boldsymbol{d}) \boldsymbol{I}+2 \mu_{s} \boldsymbol{D}(\boldsymbol{d})$, where $\boldsymbol{d}$ is the structure displacement, $\lambda_{s}, \mu_{s}>0$ are the Lamé parameters. We also introduce the fourth order elastic tensor $\mathcal{C}_{s}$ such that $\boldsymbol{T}_{S}(d)=\mathcal{C}_{S} D(d)$, see e.g. [66]. To pass from the Cauchy stress tensor $\boldsymbol{T}_{s}(\boldsymbol{d})$ to the Piola-Kirchhoff stress tensor $\widehat{T}_{s}(\widehat{d})$, we use as usual the following formula:

$$
\widehat{T}_{S}=J T_{S} F^{-T},
$$

with $J=\operatorname{det}(\boldsymbol{F})$ and where $\boldsymbol{F}=\nabla \boldsymbol{x}$ is the deformation tensor, with the gradient evaluated in the reference space coordinates and $x$ the coordinates of the points in the current configuration.

To prevent the penetration of the solid domain $\Omega_{s}$ into the rigid wall $\Gamma_{w}$, we impose that their distance $g_{C}$ is greater than a specific threshold $\varepsilon \in \mathbb{R}^{+}$, where $g_{C}: \partial \Omega_{S} \rightarrow \mathbb{R}$ is the gap function defined as

$$
g_{C}(\boldsymbol{x})=\left(\Pi_{s \rightarrow w}(\boldsymbol{x})-\boldsymbol{x}\right) \cdot \boldsymbol{n}_{w}
$$


and where we have introduced the map $\Pi_{s \rightarrow w}: \partial \Omega_{s} \rightarrow \Gamma_{w}$ defined as $\Pi_{s \rightarrow w}(\boldsymbol{x})=(x, 0)$ that projects a point from the boundary of the solid domain to the wall. Notice that $g_{C}(\boldsymbol{x})=g_{C}(\boldsymbol{X}+\widehat{\boldsymbol{d}})$, where $\boldsymbol{X}$ are the point coordinates in the reference configuration. For the sake of notation, in what follows we will write $g_{C}(\boldsymbol{d})$ instead of $g_{C}(\boldsymbol{X}+\widehat{\boldsymbol{d}})$ to highlight the dependence of $g_{C}$ on the structure displacement.

The contact region $\Gamma_{C}$ is identified as the subregion of $\partial \Omega_{s}$ such that $g_{C}=\varepsilon$. Here, a repulsive "force" (contact traction) $\lambda_{C}: \partial \Omega_{S} \rightarrow \mathbb{R}^{2}$ is prescribed to the solid:

$$
\lambda_{C}=\lambda_{C} \boldsymbol{n}_{w}+\varphi_{C} \boldsymbol{\tau}_{w},
$$

where $\lambda_{C}$ is the normal component aligned with $\boldsymbol{n}_{w}$ and $\varphi_{C}$ the tangential one, which is assumed to be zero. This leads to a contact model which is friction-less and is prescribed via an asymmetric main/secondary approach $[60,85]$, i.e. the contact conditions are prescribed only on the secondary body. In our framework, the secondary body is the solid domain $\Omega_{s}$, while we consider the wall $\Gamma_{w}$ as the rigid and fixed main body. Then, given $\varepsilon>0$, the conditions that account for the contact between the structure $\Omega_{S}$ and the wall $\Gamma_{w}$ read as follows [24]:

$$
\begin{aligned}
& g_{C} \geq \varepsilon, \quad \lambda_{C} \leq 0, \quad\left(\varepsilon-g_{C}\right) \lambda_{C}=0 \quad \text { on } \partial \Omega_{s}, \\
& \varphi_{C}=0 \quad \text { on } \partial \Omega_{s} \text {. }
\end{aligned}
$$

Notice that, to impose conditions (2.1), the (constant) normal $\boldsymbol{n}_{w}$ appearing in the definition of $g_{C}$ has to be translated and applied to $\partial \Omega_{s}$.

Remark 2.1. In condition (2.1a), the relaxation parameter $\varepsilon \geq 0$ has been introduced so that the contact arises when the gap function $g_{C}$ is equal to the threshold distance $\varepsilon$. This choice is motivated to avoid changes of topology in the discrete setting. Indeed, the non-linear nature of the contact conditions requires an iterative procedure for solving the resulting non-linear problem, and its solution fulfils the constraints only at convergence. The first inequality in Eq. (2.1a) prescribes that the distance between the solid domain and the wall has to be greater than or equal to $\varepsilon$, i.e. no-penetration of the structure in the region far at most $\varepsilon$ from the wall $\Gamma_{w}$ is allowed. The second inequality prescribes that the normal contact traction on the solid domain acts as a compression, i.e. a repulsive force. The last condition in (2.1a) ensures that at least one of the two previous inequalities is zero. Finally, condition $(2.1 \mathrm{~b})$ prescribes no friction.

The standard fluid-structure interface $\Sigma_{\text {no-slip }}$ is the portion "far away" from the contact region, defined as:

$$
\Sigma_{\text {no-slip }}=\left\{x \in \partial \Omega_{s}: g_{C}(x)>g_{\text {slip }}\right\},
$$

where $g_{\text {slip }}>\varepsilon$ is a suitable threshold distance and where we assume standard FSI conditions.

Instead, $\Sigma_{\text {slip }}$ is the portion "close" to the contact region, defined as

$$
\Sigma_{\text {slip }}=\left\{x \in \partial \Omega_{S}: \varepsilon<g_{C}(x) \leq g_{\text {slip }}\right\} .
$$


On such a portion of the interface, slip conditions are prescribed. These prescribe the standard continuity of velocities and tractions in the normal direction, whereas in the tangential direction homogeneous Neumann conditions for both fluid and structure are imposed, i.e.

$$
\begin{aligned}
& \boldsymbol{u} \cdot \boldsymbol{n}=\partial_{t} \boldsymbol{d} \cdot \boldsymbol{n}, \quad \boldsymbol{T}_{f}(\boldsymbol{u}, p) \boldsymbol{n} \cdot \boldsymbol{n}=\boldsymbol{T}_{S}(\boldsymbol{d}) \boldsymbol{n} \cdot \boldsymbol{n} \quad \text { on } \Sigma_{\text {slip }}, \\
& \boldsymbol{T}_{f}(\boldsymbol{u}, p) \boldsymbol{n} \cdot \boldsymbol{\tau}=0, \quad \boldsymbol{T}_{S}(\boldsymbol{d}) \boldsymbol{n} \cdot \boldsymbol{\tau}=0 \quad \text { on } \Sigma_{\text {slip }} .
\end{aligned}
$$

As observed, close to the contact region $\Gamma_{C}$, a thin fluid layer of size $\varepsilon$ is still present. Thus, here we apply the slip fluid-structure interface conditions in presence of contact that, due to the additional contact "force" $\lambda_{C}$, become:

$$
\begin{aligned}
& \boldsymbol{u} \cdot \boldsymbol{n}=\partial_{t} \boldsymbol{d} \cdot \boldsymbol{n}, \quad \boldsymbol{T}_{f}(\boldsymbol{u}, p) \boldsymbol{n} \cdot \boldsymbol{n}=\boldsymbol{T}_{S}(\boldsymbol{d}) \boldsymbol{n} \cdot \boldsymbol{n}-\lambda_{C} \quad \text { on } \Gamma_{C}, \\
& \boldsymbol{T}_{f}(\boldsymbol{u}, p) \boldsymbol{n} \cdot \boldsymbol{\tau}=0, \quad \boldsymbol{T}_{S}(\boldsymbol{d}) \boldsymbol{n} \cdot \boldsymbol{\tau}-\varphi_{C}=0 \quad \text { on } \Gamma_{C} .
\end{aligned}
$$

Remark 2.2. We point out that the definitions of $\Sigma_{\text {no-slip }}, \Sigma_{\text {slip }}$ and $\Gamma_{C}$ are valid also in the case of a non-convex solid domain $\Omega_{s}$, where each of these regions may be nonconnected.

The resulting contact problem in presence of FSI reads as follows: for any $t \in(0, T]$, find the fluid velocity $u$, the fluid pressure $p$, the solid displacement $d$, and the contact force $\lambda_{C}$, such that

Fluid equations:

$$
\begin{array}{ll}
\rho_{f} \partial_{t} \boldsymbol{u}+\rho_{f} \boldsymbol{u} \cdot \nabla \boldsymbol{u}-\nabla \cdot \boldsymbol{T}_{f}(\boldsymbol{u}, p)=\boldsymbol{f}_{f} & \text { in } \Omega_{f}, \\
\nabla \cdot \boldsymbol{u}=\mathbf{0} & \text { in } \Omega_{f}, \\
\boldsymbol{u}=\mathbf{0} & \text { on } \Gamma_{f} \cup \Gamma_{w} ;
\end{array}
$$

Structure equation:

$$
\rho_{s} \partial_{t t} \widehat{d}-\nabla \cdot \widehat{T}_{s}(\widehat{d})=\widehat{f}_{s} \quad \text { in } \widehat{\Omega}_{s} ;
$$

No-slip conditions at FSI interface $\Sigma_{\text {no-slip: }}$

$$
\begin{array}{ll}
\boldsymbol{u}=\partial_{t} \boldsymbol{d} & \text { on } \Sigma_{\mathrm{no}-\mathrm{slip}} \\
\boldsymbol{T}_{f}(\boldsymbol{u}, p) \boldsymbol{n}=\boldsymbol{T}_{S}(\boldsymbol{d}) \boldsymbol{n} & \text { on } \Sigma_{\mathrm{no}-\mathrm{slip}}
\end{array}
$$

Slip conditions at FSI interface $\Sigma_{\text {slip }}$ :

$$
\begin{array}{ll}
\boldsymbol{u} \cdot \boldsymbol{n}=\partial_{t} \boldsymbol{d} \cdot \boldsymbol{n} & \text { on } \Sigma_{\text {slip }} \\
\boldsymbol{T}_{f}(\boldsymbol{u}, p) \boldsymbol{n} \cdot \boldsymbol{n}=\boldsymbol{T}_{s}(\boldsymbol{d}) \boldsymbol{n} \cdot \boldsymbol{n} & \text { on } \Sigma_{\mathrm{slip}} \\
\boldsymbol{T}_{f}(\boldsymbol{u}, p) \boldsymbol{n} \cdot \boldsymbol{\tau}=0 & \text { on } \Sigma_{\mathrm{slip}} \\
\boldsymbol{T}_{S}(\boldsymbol{d}) \boldsymbol{n} \cdot \boldsymbol{\tau}=0 & \text { on } \Sigma_{\mathrm{slip}}
\end{array}
$$


Slip conditions in presence of contact on $\Gamma_{C}$ :

$$
\begin{array}{ll}
\boldsymbol{u} \cdot \boldsymbol{n}=\partial_{t} \boldsymbol{d} \cdot \boldsymbol{n} & \text { on } \Gamma_{C}, \\
\boldsymbol{T}_{f}(\boldsymbol{u}, p) \boldsymbol{n} \cdot \boldsymbol{n}=\boldsymbol{T}_{S}(\boldsymbol{d}) \boldsymbol{n} \cdot \boldsymbol{n}-\lambda_{C} & \text { on } \Gamma_{C}, \\
\boldsymbol{T}_{f}(\boldsymbol{u}, p) \boldsymbol{n} \cdot \boldsymbol{\tau}=0 & \text { on } \Gamma_{C} \\
\boldsymbol{T}_{S}(\boldsymbol{d}) \boldsymbol{n} \cdot \boldsymbol{\tau}-\varphi_{C}=0 & \text { on } \Gamma_{C}
\end{array}
$$

Contact conditions:

$$
\begin{array}{ll}
g_{C} \geq \varepsilon, \quad \lambda_{C} \leq 0, \quad\left(\varepsilon-g_{C}\right) \lambda_{C}=0 & \text { on } \partial \Omega_{S}, \\
\varphi_{C}=0 & \text { on } \partial \Omega_{s},
\end{array}
$$

where $\rho_{f}$ and $\rho_{s}$ are the fluid and structure densities and $f_{f}$ and $f_{s}$ the forcing terms. Problem (2.3) is supplemented with (regular enough) initial conditions $\boldsymbol{u}(\boldsymbol{x}, 0)=\boldsymbol{u}^{0}(\boldsymbol{x})$ in $\Omega_{f}(0), \widehat{d}(x, 0)=\widehat{d}^{0}(x)$ in $\widehat{\Omega}_{s}$, and $\partial_{t} \widehat{d}(x, 0)=\widehat{v}^{0}(x)$ in $\widehat{\Omega}_{s}$.

\section{Numerical discretization}

In this section, we present the semi-discrete PolyDG formulation associated to the weak form of problem (2.3) (Section 3.1), then we discuss the time discretization and the treatment of the non-linearities and report the corresponding fully discrete formulation (Section 3.2) and finally we describe how to extend the numerical formulation in the case of two deformable structures (Section 3.3).

\subsection{Polygonal Discontinuous Galerkin approximation}

We introduce the background mesh $\mathcal{T}_{h}$ and solid mesh $\mathcal{T}_{s, h}$ that cover the entire fixed domain $\Omega$ and the structure domain $\Omega_{s}$, respectively, see Fig. 2 (top-left). Notice that, while $\mathcal{T}_{h}$ is fixed in time, $\mathcal{T}_{s, h}$ depends on time. We point out that the elements belonging to $\mathcal{T}_{h}$ and $\mathcal{T}_{s, h}$ may be polygonal elements of arbitrary shape. The intersection of the background and structure meshes induces a set of background elements that are crossed by the boundary of $\mathcal{T}_{s, h}$, see Fig. 2 (bottom-left), defined as:

$$
\mathcal{G}_{h}=\left\{K \in \mathcal{T}_{h}: \varnothing \neq K \cap \Omega_{s} \subsetneq K\right\} .
$$

Each element $K \in \mathcal{G}_{h}$ can be partitioned into elements of arbitrary shape $\left\{P_{K}^{j}\right\}_{j}$ resulting from the intersection with the boundary of the structure mesh, see Fig. 2 (bottom-right). The computational grid $\mathcal{T}_{f, h}$ associated with the fluid domain $\Omega_{f}$ is obtained as the union of the elements of $\mathcal{T}_{h}$ that are not intersected by the structure domain, plus the cut elements that are partially covered by the structure domain, i.e.,

$$
\mathcal{T}_{f, h}=\left\{K \in \mathcal{T}_{h}: K \cap K_{s}=\varnothing \forall K_{s} \in \mathcal{T}_{s, h}\right\} \cup\left\{P_{K}^{j} \subset K \in \mathcal{G}_{h}: P_{K}^{j} \cap \Omega_{f} \neq \varnothing\right\},
$$



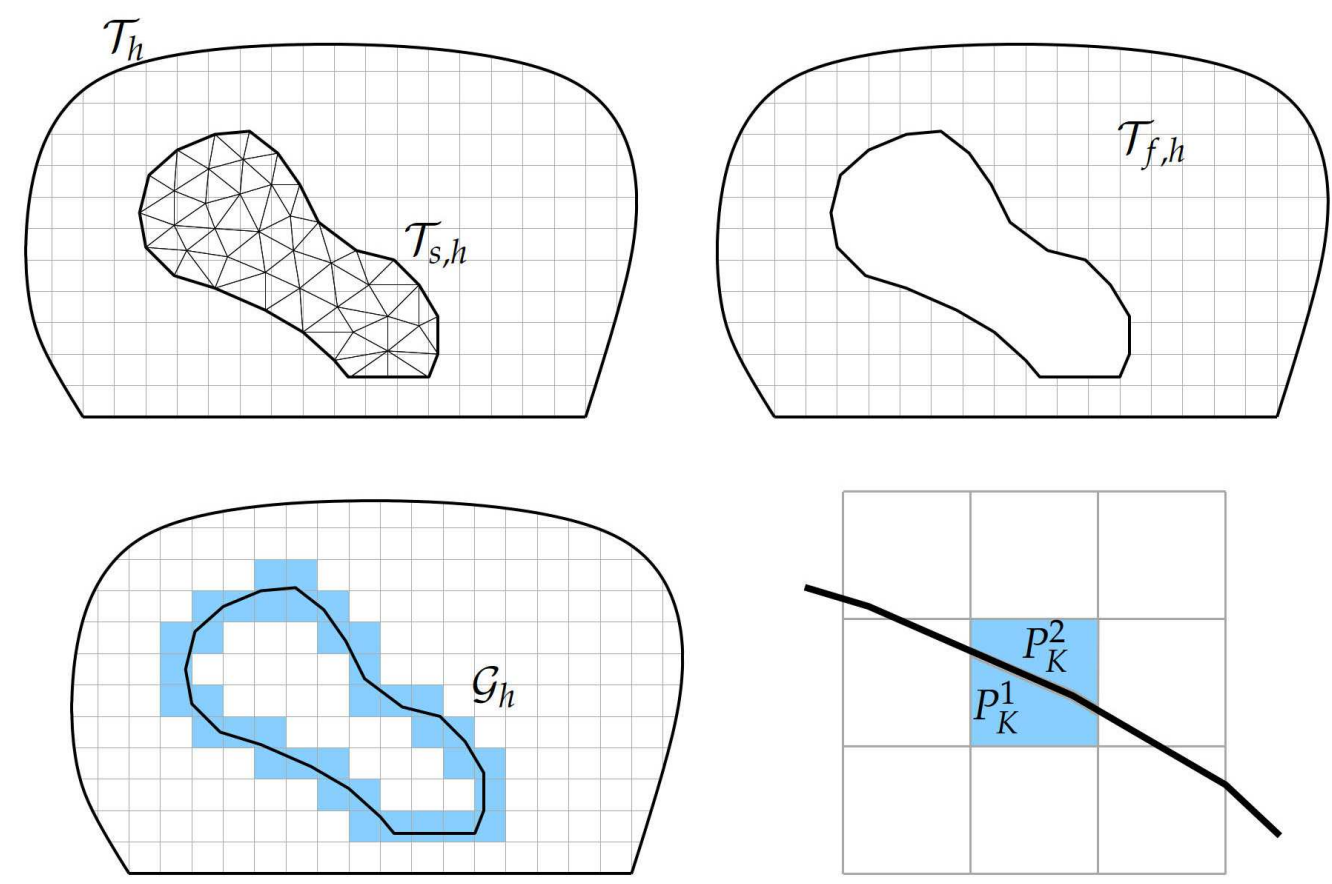

Figure 2: Top-left: background mesh $\mathcal{T}_{h}$ (gray) and structure mesh $\mathcal{T}_{s, h}$ (black). Top right: fluid mesh $\mathcal{T}_{f, h}$. Bottom-left: elements belonging to the set $\mathcal{G}_{h}$ defined as in (3.1) (blue). Bottom-right: detail of an element $K \in \mathcal{G}_{h}$ that is cut into $P_{K}^{1}$ and $P_{K}^{2}$ (blue).

see Fig. 2 (top-right).

In what follows, we assume that $\mu_{f}$ and $\mathcal{C}_{s}$ are piecewise constant over the mesh.

We denote by $\mathcal{F}_{i, h}, i=\{f$,s,no-slip, slip, $C\}$, the set of one-dimensional edges composing $\mathcal{T}_{f, h}, \mathcal{T}_{s, h}, \Sigma_{\text {no-slip }}, \Sigma_{\text {slip }}$ and $\Gamma_{C}$, respectively, and $\mathcal{F}_{\Sigma, h}=\mathcal{F}_{\text {no-slip }, h} \cup \mathcal{F}_{\text {slip }, h} \cup \mathcal{F}_{C, h}$.

In what follows, we introduce the DG Finite Elements spaces of order $\ell$, defined as

$$
\begin{aligned}
& \boldsymbol{V}_{f, h}^{\ell}(t)=\left\{\boldsymbol{v}(t) \in\left[L^{2}\left(\Omega_{f}(t)\right)\right]^{2}:\left.\boldsymbol{v}\right|_{K} \in\left[\mathcal{P}^{\ell}(K)\right]^{2} \forall K \in \mathcal{T}_{f, h}(t)\right\}, \\
& Q_{h}^{\ell}(t)=\left\{q(t) \in L^{2}\left(\Omega_{f}(t)\right):\left.q\right|_{K} \in \mathcal{P}^{\ell}(K) \forall K \in \mathcal{T}_{f, h}(t)\right\}, \\
& \boldsymbol{V}_{s, h}^{\ell}=\left\{\boldsymbol{w} \in\left[L^{2}\left(\widehat{\Omega}_{s}\right)\right]^{2}:\left.\boldsymbol{w}\right|_{K} \in\left[\mathcal{P}^{\ell}(K)\right]^{2} \forall K \in \widehat{\mathcal{T}}_{s, h}\right\},
\end{aligned}
$$

where $\widehat{\mathcal{T}}_{s, h}$ is the structure mesh in the reference (initial) configuration and $\mathcal{P}^{\ell}(K)$ denotes the space of polynomials obtained over the polygon $K$ of total degree at most $\ell \geq 1$. Notice that the polynomial space $\mathcal{P}^{\ell}(K)$ is based on a modal expansion that allows to easily build high-order DG spaces. Moreover, the shape functions and the degrees of freedom are directly generated on the (polygonal) physical element $K$ with the "bounding box" technique, as described in [32]. This simplifies the implementation with respect to the Finite Elements methods. Indeed, for the XFEM the shape functions and the degrees of freedom are defined on the original triangular/quadrilateral element $K$, yielding the assembling of the local matrices corresponding to $\left\{P_{K}^{j}\right\}_{j}$ more tricky. 
Remark 3.1. Notice that in the present work, the fluid-structure interface is represented as a piecewise linear interface, even if the polynomial approximation degree $\ell$ may be greater than one. This choice is motivated to simplify the computation of the intersections between the fluid and structure meshes. A possible extension to a high-order isoparametric mapping with Cut-FEM is proposed in [63] for elliptic problems, while in [7] a high-order isoparametric representation of the interface with the DG methods is proposed for elliptic problems on surfaces.

Remark 3.2. Notice that the total degree $\ell$ for the polynomials in the space $\mathcal{P}^{\ell}$ can be in general chosen differently for the fluid velocity, fluid pressure and solid displacement. To ease the exposition, in what follows and in the numerical experiments we consider the same order for all the three spaces.

On any interior edge $F \in \mathcal{F}_{i, h}, i=\{f, s$,no-slip, slip, $C\}$, and for regular enough vectorvalued and symmetric tensor-valued functions $v$ and $T$, respectively, we define the average and jump operators as

$$
\{T\}=\frac{1}{2}\left(T^{+}+T^{-}\right), \quad \llbracket v \rrbracket=v^{+} \odot n^{+}+v^{-} \odot n^{-},
$$

where $v^{ \pm}$and $T^{ \pm}$denote the traces of $v$ and $T$ on any edge $F$ between elements $K^{ \pm}$and where $\boldsymbol{v} \odot \boldsymbol{n}=\left(\boldsymbol{v} \boldsymbol{n}^{T}+\boldsymbol{n} \boldsymbol{v}^{T}\right) / 2$. Notice that $\llbracket \boldsymbol{v} \rrbracket$ is a symmetric tensor-valued function. On a boundary edge $F \in \mathcal{F}_{f, h}$ where an homogeneous Dirichlet condition is prescribed, we set analogously

$$
\{T\}=T, \quad \llbracket v \rrbracket=v \odot n .
$$

We also introduce the $L^{2}$-inner products over the domain $Z$ and edges $\mathcal{F}_{i, h}$ with the shorthand notation $(\cdot, \cdot)_{Z}$ and $(\cdot, \cdot)_{\mathcal{F}_{i, h}}$, respectively.

The semi-discrete PolyDG approximation reads as follows: given $\delta \in[0,1], \sigma_{f} \in L^{\infty}\left(\mathcal{F}_{f, h}\right)$, $\widehat{\sigma}_{s} \in L^{\infty}\left(\widehat{\mathcal{F}}_{s, h}\right), \sigma_{\Sigma} \in L^{\infty}\left(\mathcal{F}_{\Sigma, h}\right), \sigma_{C} \in L^{\infty}\left(\mathcal{F}_{C, h}\right), f_{f} \in\left[L^{2}\left(\Omega_{f}\right)\right]^{2}$ and $\widehat{f}_{s} \in\left[L^{2}\left(\widehat{\Omega}_{s}\right)\right]^{2}$, for any $t \in(0, T]$, find $\left(\boldsymbol{u}_{h}(t), p_{h}(t), \widehat{\boldsymbol{d}}_{h}(t)\right) \in \boldsymbol{V}_{f, h}^{\ell}(t) \times Q_{h}^{\ell}(t) \times \boldsymbol{V}_{s, h}^{\ell}$, such that

$$
\begin{aligned}
A_{f, h}\left(\boldsymbol{u}_{h}, \boldsymbol{u}_{h}, p_{h} ; \boldsymbol{v}_{h}, q_{h}\right)+A_{s, h}\left(\widehat{\boldsymbol{d}}_{h}, \widehat{\boldsymbol{w}}_{h}\right) & +A_{\text {no-slip }, h}\left(\boldsymbol{u}_{h}, p_{h}, \boldsymbol{d}_{h} ; \boldsymbol{v}_{h}, q_{h}, \boldsymbol{w}_{h}\right) \\
& +A_{\text {slip }, h}\left(\boldsymbol{u}_{h}, p_{h}, \boldsymbol{d}_{h} ; \boldsymbol{v}_{h}, q_{h}, \boldsymbol{w}_{h}\right) \\
& +A_{C, h}\left(\boldsymbol{u}_{h}, p_{h}, \boldsymbol{d}_{h} ; \boldsymbol{v}_{h}, q_{h}, \boldsymbol{w}_{h}\right)=F_{h}\left(\boldsymbol{v}_{h}, \boldsymbol{w}_{h}\right),
\end{aligned}
$$

for all $\left(\boldsymbol{v}_{h}, q_{h}, \widehat{\boldsymbol{w}}_{h}\right) \in \boldsymbol{V}_{f, h}^{\ell}(t) \times Q_{h}^{\ell}(t) \times \boldsymbol{V}_{s, h}^{\ell}$. Here, we have set

$$
\begin{aligned}
A_{f, h}\left(\boldsymbol{u}_{h}, \boldsymbol{u}_{h}, p_{h} ; \boldsymbol{v}_{h}, \boldsymbol{q}_{h}\right)= & \rho_{f}\left(\partial_{t} \boldsymbol{u}_{h}, \boldsymbol{v}_{h}\right)_{\Omega_{f}}+a_{f}\left(\boldsymbol{u}_{h}, \boldsymbol{v}_{h}\right)+b\left(p_{h}, \boldsymbol{v}_{h}\right)-b\left(q_{h}, \boldsymbol{u}_{h}\right)+c\left(\boldsymbol{u}_{h}, \boldsymbol{u}_{h}, \boldsymbol{v}_{h}\right) \\
& -\left(\left\{\boldsymbol{T}_{f}\left(\boldsymbol{u}_{h}, p_{h}\right)\right\}, \llbracket \boldsymbol{v}_{h} \rrbracket\right)_{\mathcal{F}_{f, h}}-\left(\llbracket \boldsymbol{u}_{h} \rrbracket,\left\{\boldsymbol{T}_{f}\left(\boldsymbol{v}_{h},-q_{h}\right)\right\}\right)_{\mathcal{F}_{f, h}} \\
& +\left(\sigma_{f} \llbracket \boldsymbol{u}_{h} \rrbracket, \llbracket \boldsymbol{v}_{h} \rrbracket\right)_{\mathcal{F}_{f, h}}+s_{h}\left(p_{h}, q_{h}\right) ;
\end{aligned}
$$




$$
\begin{aligned}
& A_{s, h}\left(\widehat{\boldsymbol{d}}_{h}, \widehat{\boldsymbol{w}}_{h}\right)=\rho_{s}\left(\partial_{t t} \widehat{\boldsymbol{d}}_{h}, \widehat{\boldsymbol{w}}_{h}\right)_{\widehat{\Omega}_{s}}+a_{s}\left(\widehat{\boldsymbol{d}}_{h}, \widehat{\boldsymbol{w}}_{h}\right) \\
& -\left(\left\{\widehat{\boldsymbol{T}}_{\mathcal{S}}\left(\widehat{\boldsymbol{d}}_{h}\right)\right\}, \llbracket \widehat{\boldsymbol{w}}_{h} \rrbracket\right)_{\widehat{\mathcal{F}}_{s, h}}-\left(\llbracket \widehat{\boldsymbol{d}}_{h} \rrbracket,\left\{\widehat{\boldsymbol{T}}_{\mathcal{S}}\left(\widehat{\boldsymbol{w}}_{h}\right)\right\}\right)_{\widehat{\mathcal{F}}_{s, h}} \\
& +\left(\widehat{\sigma}_{s} \llbracket \widehat{\boldsymbol{d}}_{h} \rrbracket, \llbracket \widehat{\boldsymbol{w}}_{h} \rrbracket\right)_{\widehat{\mathcal{F}}_{s, h}} ; \\
& A_{\text {no-slip }, h}\left(\boldsymbol{u}_{h}, p_{h}, \boldsymbol{d}_{h} ; \boldsymbol{v}_{h}, q_{h}, \boldsymbol{w}_{h}\right)=-\left(\delta \boldsymbol{T}_{f}\left(\boldsymbol{u}_{h}, p_{h}\right) \boldsymbol{n}+(1-\delta) \boldsymbol{T}_{s}\left(\boldsymbol{d}_{h}\right) \boldsymbol{n}, \boldsymbol{v}_{h}-\boldsymbol{w}_{h}\right)_{\mathcal{F}_{\text {no-slip }, h}} \\
& -\left(\boldsymbol{u}_{h}-\partial_{t} \boldsymbol{d}_{h}, \delta \boldsymbol{T}_{f}\left(\boldsymbol{v}_{h},-q_{h}\right) \boldsymbol{n}+(1-\delta) \boldsymbol{T}_{S}\left(\boldsymbol{w}_{h}\right) \boldsymbol{n}\right)_{\mathcal{F}_{\text {no-slip }, h}} \\
& +\left(\sigma_{\Sigma}\left(\boldsymbol{u}_{h}-\partial_{t} \boldsymbol{d}_{h}\right), \boldsymbol{v}_{h}-\boldsymbol{w}_{h}\right)_{\mathcal{F}_{\text {no-slip }, h}} \\
& A_{\text {slip }, h}\left(\boldsymbol{u}_{h}, p_{h}, \boldsymbol{d}_{h} ; \boldsymbol{v}_{h}, q_{h}, \boldsymbol{w}_{h}\right)=-\left(\delta \boldsymbol{T}_{f}\left(\boldsymbol{u}_{h}, p_{h}\right) \boldsymbol{n} \cdot \boldsymbol{n}+(1-\delta) \boldsymbol{T}_{s}\left(\boldsymbol{d}_{h}\right) \boldsymbol{n} \cdot \boldsymbol{n}, \boldsymbol{v}_{h} \cdot \boldsymbol{n}-\boldsymbol{w}_{h} \cdot \boldsymbol{n}\right)_{\mathcal{F}_{\text {slip }, h}} \\
& -\left(\boldsymbol{u}_{h} \cdot \boldsymbol{n}-\partial_{t} \boldsymbol{d}_{h} \cdot \boldsymbol{n}, \delta \boldsymbol{T}_{f}\left(\boldsymbol{v}_{h},-q_{h}\right) \boldsymbol{n} \cdot \boldsymbol{n}+(1-\delta) \boldsymbol{T}_{S}\left(\boldsymbol{w}_{h}\right) \boldsymbol{n} \cdot \boldsymbol{n}\right)_{\mathcal{F}_{\text {slip }, h}} \\
& +\left(\sigma_{\Sigma}\left(\boldsymbol{u}_{h} \cdot \boldsymbol{n}-\partial_{t} \boldsymbol{d}_{h} \cdot \boldsymbol{n}\right), \boldsymbol{v}_{h} \cdot \boldsymbol{n}-\boldsymbol{w}_{h} \cdot \boldsymbol{n}\right)_{\mathcal{F}_{\text {slip }, h}} ; \\
& -\left(\boldsymbol{u}_{h} \cdot \boldsymbol{n}-\partial_{t} \boldsymbol{d}_{h} \cdot \boldsymbol{n}, \delta \boldsymbol{T}_{f}\left(\boldsymbol{v}_{h},-q_{h}\right) \boldsymbol{n} \cdot \boldsymbol{n}+(1-\delta) \boldsymbol{T}_{S}\left(\boldsymbol{w}_{h}\right) \boldsymbol{n} \cdot \boldsymbol{n}\right)_{\mathcal{F}_{C, h}} \\
& +\left(\sigma_{\Sigma}\left(\boldsymbol{u}_{h} \cdot \boldsymbol{n}-\partial_{t} \boldsymbol{d}_{h} \cdot \boldsymbol{n}\right), \boldsymbol{v}_{h} \cdot \boldsymbol{n}-\boldsymbol{w}_{h} \cdot \boldsymbol{n}\right)_{\mathcal{F}_{C, h}} \\
& -\left(\sigma_{C} g_{C}\left(\boldsymbol{d}_{h}\right), \boldsymbol{w}_{h} \cdot \boldsymbol{n}\right)_{\mathcal{F}_{C, h}} \\
& -\left(\delta\left(\boldsymbol{T}_{f}\left(\boldsymbol{u}_{h}, p_{h}\right) \boldsymbol{n}-\boldsymbol{T}_{S}\left(\boldsymbol{d}_{h}\right) \boldsymbol{n}\right) \cdot \boldsymbol{n}, \boldsymbol{w}_{h} \cdot \boldsymbol{n}\right)_{\mathcal{F}_{C, h}} \\
& -\left((1-\delta)\left(\boldsymbol{T}_{f}\left(\boldsymbol{u}_{h}, p_{h}\right) \boldsymbol{n}-\boldsymbol{T}_{S}\left(\boldsymbol{d}_{h}\right) \boldsymbol{n}\right) \cdot \boldsymbol{n}, \boldsymbol{v}_{h} \cdot \boldsymbol{n}\right)_{\mathcal{F}_{C, h}} ; \\
& F_{h}\left(\boldsymbol{v}_{h}, \boldsymbol{w}_{h}\right)=\left(\boldsymbol{f}_{f}, \boldsymbol{v}_{h}\right)_{\Omega_{f}}+\left(\widehat{\boldsymbol{f}}_{s}, \widehat{\boldsymbol{w}}_{h}\right)_{\widehat{\Omega}_{s}}-\left(\sigma_{C} \varepsilon, \boldsymbol{w}_{h} \cdot \boldsymbol{n}\right)_{\mathcal{F}_{C, h}} .
\end{aligned}
$$

In (3.5)-(3.6) the bilinear forms $a_{f}: V_{f, h}^{\ell} \times V_{f, h}^{\ell} \rightarrow \mathbb{R}, b: Q_{h}^{\ell} \times V_{f, h}^{\ell} \rightarrow \mathbb{R}$ and $a_{s}: V_{s, h}^{\ell} \times V_{s, h}^{\ell} \rightarrow \mathbb{R}$ are defined as

$$
\begin{aligned}
& a_{f}(\boldsymbol{u}, \boldsymbol{v})=2 \mu_{f}(\boldsymbol{D}(\boldsymbol{u}), \boldsymbol{D}(\boldsymbol{v}))_{\Omega_{f^{\prime}}} \quad b(p, \boldsymbol{u})=-(p, \nabla \cdot \boldsymbol{u})_{\Omega_{f}{ }^{\prime}} \\
& a_{s}(\widehat{\boldsymbol{d}}, \widehat{\boldsymbol{w}})=\left(\widehat{\boldsymbol{T}}_{s}(\widehat{\boldsymbol{d}}), \nabla \widehat{\boldsymbol{w}}\right)_{\widehat{\Omega}_{s}}=\lambda_{s}(\nabla \cdot \widehat{\boldsymbol{d}}, \nabla \cdot \widehat{\boldsymbol{w}})_{\widehat{\Omega}_{s}}+2 \mu_{s}(\boldsymbol{D}(\widehat{\boldsymbol{d}}), \boldsymbol{D}(\widehat{\boldsymbol{w}}))_{\widehat{\Omega}_{s}{ }^{\prime}}
\end{aligned}
$$

whereas the trilinear form $c: V_{f, h}^{\ell} \times V_{f, h}^{\ell} \times V_{f, h}^{\ell} \rightarrow \mathbb{R}$ is defined as in [42] and is given by

$$
\begin{aligned}
c(\boldsymbol{w}, \boldsymbol{u}, \boldsymbol{v})= & \rho_{f}(\boldsymbol{w} \cdot \nabla \boldsymbol{u}, \boldsymbol{v})_{\Omega_{f}}+\frac{\rho_{f}}{2}(\nabla \cdot \boldsymbol{w}, \boldsymbol{u} \cdot \boldsymbol{v})_{\Omega_{f}} \\
& -\rho_{f}(\{\{\boldsymbol{w}\}\} \cdot \boldsymbol{n}, \llbracket \boldsymbol{u} \rrbracket \cdot\{\{\boldsymbol{v}\}\})_{\mathcal{F}_{f, h}}-\frac{\rho_{f}}{2}(\llbracket \boldsymbol{w} \rrbracket \cdot \boldsymbol{n},\{\{\boldsymbol{u} \cdot \boldsymbol{v}\}\})_{\mathcal{F}_{f, h}} .
\end{aligned}
$$

In (3.5), $s_{h}: Q_{h}^{\ell} \times Q_{h}^{\ell} \rightarrow \mathbb{R}$ is the Interior Penalty term for pressure stabilization [42] with parameter $\gamma_{p}$, that allows us to use equal order elements for velocity and pressure. A 
theoretical study on possible pairs of spaces that satisfy the inf-sup condition for the proposed method on arbitrary polygonal/polyhedral meshes is under investigation. See $[3,22,43]$ and $[5,16,17,33]$ for a study of the Stokes problem on general meshes with the Hybrid High-Order and the Virtual Element methods, respectively.

In (3.5)-(3.9), $\sigma_{f}, \widehat{\sigma}_{s}, \sigma_{\Sigma}, \sigma_{C}$ are the four positive penalty functions which are piecewise constant over the edges $F$ belonging to $\mathcal{F}_{i, h}, i=\{f, s, \Sigma, C\}$. Their definition over $F$ is as follows:

$$
\begin{array}{ll}
\left.\sigma_{f}\right|_{F}=\gamma_{f} \max _{K^{+}, K^{-}}\left\{\frac{2 \ell^{2} \mu_{f}}{h_{K}}\right\}, & \bar{F}=\partial \bar{K}^{+} \cap \partial \bar{K}^{-} \in \mathcal{F}_{f, h}, \\
\left.\widehat{\sigma}_{s}\right|_{F}=\gamma_{s} \max _{K^{+}, K^{-}}\left\{\frac{\ell^{2} \overline{\mathcal{C}}_{s, K}}{h_{K}}\right\}, & \bar{F}=\partial \bar{K}^{+} \cap \partial \bar{K}^{-} \in \widehat{\mathcal{F}}_{s, h}, \\
\left.\sigma_{\Sigma}\right|_{F}=\gamma_{\Sigma} \max _{K^{+}, K^{-}}\left\{\frac{\ell^{2}}{h_{K}}\left(2 \delta \mu_{f}+(1-\delta) \overline{\mathcal{C}}_{s, K}\right)\right\}, & \bar{F}=\partial \bar{K}^{+} \cap \partial \bar{K}^{-} \in \mathcal{F}_{\Sigma, h}, \\
\left.\sigma_{C}\right|_{F}=\gamma_{C} \frac{\ell^{2}}{h_{K}} \overline{\mathcal{C}}_{s, K} & F \in \mathcal{F}_{C, h} .
\end{array}
$$

Here, $h_{K}$ is the diameter of the element $K$ and $\overline{\mathcal{C}}_{s, K}=\left\|\left.\mathcal{C}_{s}\right|_{K}\right\|_{\ell^{2}}$. Moreover, $\gamma_{f}, \gamma_{s}, \gamma_{\Sigma}$ and $\gamma_{C}$ are positive constants that will be chosen later on.

Remark 3.3. The parameter $\delta$ arises from the weighted average operator at the fluidstructure interface in the consistency and symmetry terms. This parameter allows to unbalance the contributions of the fluid and solid stresses at the interface. In [11], in the case of a FSI problem without contact, the stability analysis shows that the PolyDG method is stable for the choice $\delta=1$, while there is no theoretical result for $\delta \neq 1$. At the best of our knowledge, there is no stability analysis for the PolyDG method in the case of a FSI problem with contact; the stability analysis for this case is under investigation.

Remark 3.4. Notice that the last two terms in (3.9) arise as a consequence of consistency. Indeed, after the integration by parts in the fluid and solid bilinear forms, a term depending on $\lambda_{C}$ appears on $\Gamma_{C}$ which can be replaced by exploiting equations (2.3l) and (2.3p). Thus, in fact the contact force $\lambda_{C}$ does not appear as an explicit unknown of the discrete problem.

Remark 3.5. We point out that in the PolyDG formulation (3.4) the inequalities appearing in Eq. (2.3o) are "hidden" in the definition of $\Gamma_{C}$. Indeed, if $g_{C}(x)>\varepsilon$ for all $x$, then $\Gamma_{C}=\varnothing$ and the term $A_{C, h}$ is zero and does not introduce any force to the system, i.e. $\lambda_{C}=0$; otherwise, if $g_{C}(x)=\varepsilon$ for some points $x \in \partial \Omega_{s}$, we have $\Gamma_{C}=\left\{x: g_{C}(x)=\varepsilon\right\}$ and the term $A_{C, h}$ prescribes a repulsive force $\lambda_{C}<0$ on $\Gamma_{C}$.

\subsection{Time discretization}

Let $\Delta t$ be the constant time discretization parameter, let $t^{n}=n \Delta t, n \geq 0$, be the $n-$ th time step, and, given the unknown $z(t)$, let $z^{n}$ be the approximation of $z\left(t^{n}\right)$. The same 
notation is used for domains and spaces.

Given $r \in \mathbb{N}^{+}$, to discretize in time the semi-discrete problem (3.4), we apply a Backward Difference Formula (BDF) scheme [55] of order $r$ both for the fluid and the solid subproblems. We introduce the BDF scheme for approximating the time derivatives, i.e.

$$
\begin{aligned}
& \partial_{t} z\left(t^{n}\right) \approx \frac{\beta_{0}}{\Delta t} z^{n}-\widetilde{z}^{n}, \quad \widetilde{z}^{n}=\sum_{i=1}^{r} \frac{\beta_{i}}{\Delta t} z^{n-i}, \\
& \partial_{t t} z\left(t^{n}\right) \approx \frac{\zeta_{0}}{\Delta t^{2}} z^{n}-\widetilde{z}^{n}, \quad \widetilde{z}^{n}=\sum_{i=1}^{r} \frac{\zeta_{i}}{\Delta t^{2}} z^{n-i},
\end{aligned}
$$

where $\beta_{i}$ and $\zeta_{i}$, for $i=0, \cdots, r$, are the coefficients of the BDF scheme for the discretization of the first and second time derivatives, respectively, see e.g. [55].

The fully-discrete PolyDG approximation reads as follows: given $\delta \in[0,1], \sigma_{f} \in$ $L^{\infty}\left(\mathcal{F}_{f, h}^{n}\right), \widehat{\sigma}_{s} \in L^{\infty}\left(\widehat{\mathcal{F}}_{s, h}\right), \sigma_{\Sigma} \in L^{\infty}\left(\mathcal{F}_{\Sigma, h}^{n}\right), \sigma_{C} \in L^{\infty}\left(\mathcal{F}_{C, h}^{n}\right), f_{f} \in\left[L^{2}\left(\Omega_{f}^{n}\right)\right]^{2}$ and $\widehat{f}_{s} \in\left[L^{2}\left(\widehat{\Omega}_{s}\right)\right]^{2}$, for $n>0$, find $\left(\boldsymbol{u}_{h}^{n}, p_{h^{n}}^{n} \widehat{\boldsymbol{d}}_{h}^{n}\right) \in \boldsymbol{V}_{f, h}^{\ell, n} \times Q_{h}^{\ell, n} \times \boldsymbol{V}_{s, h^{\prime}}^{\ell}$, such that

$$
\begin{aligned}
A_{f, h}^{n}\left(\boldsymbol{u}_{h}^{n}, \boldsymbol{u}_{h}^{n}, p_{h}^{n} ; \boldsymbol{v}_{h}, q_{h}\right)+A_{s, h}^{n}\left(\widehat{\boldsymbol{d}}_{h}^{n}, \widehat{\boldsymbol{w}}_{h}\right) & +A_{\text {no-slip }, h}^{n}\left(\boldsymbol{u}_{h}^{n}, p_{h}^{n}, \boldsymbol{d}_{h}^{n} ; \boldsymbol{v}_{h}, q_{h}, \boldsymbol{w}_{h}\right) \\
& +A_{\text {slip }, h}^{n}\left(\boldsymbol{u}_{h}^{n}, p_{h}^{n}, \boldsymbol{d}_{h}^{n} ; \boldsymbol{v}_{h}, q_{h}, \boldsymbol{w}_{h}\right) \\
& +A_{C, h}^{n}\left(\boldsymbol{u}_{h}^{n}, p_{h}^{n}, \boldsymbol{d}_{h}^{n} ; \boldsymbol{v}_{h}, q_{h}, \boldsymbol{w}_{h}\right)=F_{h}^{n}\left(\boldsymbol{v}_{h}, \boldsymbol{w}_{h}\right),
\end{aligned}
$$

for all $\left(\boldsymbol{v}_{h}, q_{h}, \widehat{\boldsymbol{w}}_{h}\right) \in \boldsymbol{V}_{f, h}^{\ell, n} \times Q_{h}^{\ell, n} \times \boldsymbol{V}_{s, h}^{\ell}$. Here, we have defined

$$
\begin{aligned}
A_{f, h}^{n}\left(\boldsymbol{u}_{h}^{n}, \boldsymbol{u}_{h}^{n}, p_{h}^{n} ; \boldsymbol{v}_{h}, q_{h}\right)= & \rho_{f}\left(\frac{\beta_{0}}{\Delta t} \boldsymbol{u}_{h}^{n}, \boldsymbol{v}_{h}\right)_{\Omega_{f}^{n}}+a_{f}^{n}\left(\boldsymbol{u}_{h}^{n}, \boldsymbol{v}_{h}\right) \\
& +b^{n}\left(p_{h}^{n}, \boldsymbol{v}_{h}\right)-b^{n}\left(q_{h}, \boldsymbol{u}_{h}^{n}\right)+c^{n}\left(\boldsymbol{u}_{h}^{n}, \boldsymbol{u}_{h}^{n}, \boldsymbol{v}_{h}\right) \\
& -\left(\left\{\boldsymbol{T}_{f}\left(\boldsymbol{u}_{h}^{n}, p_{h}^{n}\right)\right\}, \llbracket \boldsymbol{v}_{h} \rrbracket\right)_{\mathcal{F}_{f, h}^{n}}-\left(\llbracket \boldsymbol{u}_{h}^{n} \rrbracket,\left\{\boldsymbol{T}_{f}\left(\boldsymbol{v}_{h},-q_{h}\right)\right\}\right)_{\mathcal{F}_{f, h}^{n}} \\
& +\left(\sigma_{f} \llbracket \boldsymbol{u}_{h}^{n} \rrbracket, \llbracket \boldsymbol{v}_{h} \rrbracket\right)_{\mathcal{F}_{f, h}^{n}}+s_{h}^{n}\left(p_{h}^{n}, q_{h}\right) ; \\
A_{s, h}^{n}\left(\widehat{\boldsymbol{d}}_{h}^{n}, \widehat{\boldsymbol{w}}_{h}\right)= & \rho_{s}\left(\frac{\zeta_{0}}{\Delta t^{2}} \widehat{\boldsymbol{d}}_{h}^{n}, \widehat{\boldsymbol{w}}_{h}\right)_{\widehat{\Omega}_{s}}+a_{s}\left(\widehat{\boldsymbol{d}}_{h}^{n}, \widehat{\boldsymbol{w}}_{h}\right) \\
& -\left(\left\{\widehat{\boldsymbol{T}}_{s}\left(\widehat{\boldsymbol{d}}_{h}^{n}\right)\right\}, \llbracket \widehat{\boldsymbol{w}}_{h} \rrbracket\right)_{\widehat{\mathcal{F}}_{s, h}}-\left(\llbracket \widehat{\boldsymbol{d}}_{h}^{n} \rrbracket,\left\{\widehat{\boldsymbol{T}}_{s}\left(\widehat{\boldsymbol{w}}_{h}\right)\right\}\right)_{\widehat{\mathcal{F}}_{s, h}} \\
& +\left(\widehat{\sigma}_{s} \llbracket \widehat{\boldsymbol{d}}_{h}^{n} \rrbracket, \llbracket \widehat{\boldsymbol{w}}_{h} \rrbracket\right)_{\widehat{\mathcal{F}}_{s, h} ;}
\end{aligned}
$$




$$
\begin{aligned}
& A_{\text {no-slip }, h}^{n}\left(\boldsymbol{u}_{h}^{n}, p_{h}^{n}, \boldsymbol{d}_{h}^{n} ; \boldsymbol{v}_{h}, q_{h}, \boldsymbol{w}_{h}\right)=-\left(\delta \boldsymbol{T}_{f}\left(\boldsymbol{u}_{h}^{n}, p_{h}^{n}\right) \boldsymbol{n}+(1-\delta) \boldsymbol{T}_{S}\left(\boldsymbol{d}_{h}^{n}\right) \boldsymbol{n}, \boldsymbol{v}_{h}-\boldsymbol{w}_{h}\right)_{\mathcal{F}_{\text {no-slip }, h}^{n}} \\
& -\left(\boldsymbol{u}_{h}^{n}-\frac{\beta_{0}}{\Delta t} \boldsymbol{d}_{h}^{n}, \delta \boldsymbol{T}_{f}\left(\boldsymbol{v}_{h},-q_{h}\right) \boldsymbol{n}+(1-\delta) \boldsymbol{T}_{S}\left(\boldsymbol{w}_{h}\right) \boldsymbol{n}\right)_{\mathcal{F}_{\text {no-slip }, h}^{n}} \\
& +\left(\sigma_{\Sigma}\left(\boldsymbol{u}_{h}^{n}-\frac{\beta_{0}}{\Delta t} \boldsymbol{d}_{h}^{n}\right), \boldsymbol{v}_{h}-\boldsymbol{w}_{h}\right)_{\mathcal{F}_{\text {no-slip }, h}^{n}} ; \\
& A_{\text {slip }, h}^{n}\left(\boldsymbol{u}_{h}^{n}, p_{h}^{n}, \boldsymbol{d}_{h}^{n} ; \boldsymbol{v}_{h}, q_{h}, \boldsymbol{w}_{h}\right)=-\left(\delta \boldsymbol{T}_{f}\left(\boldsymbol{u}_{h}^{n}, p_{h}^{n}\right) \boldsymbol{n} \cdot \boldsymbol{n}+(1-\delta) \boldsymbol{T}_{S}\left(\boldsymbol{d}_{h}^{n}\right) \boldsymbol{n} \cdot \boldsymbol{n}, \boldsymbol{v}_{h} \cdot \boldsymbol{n}-\boldsymbol{w}_{h} \cdot \boldsymbol{n}\right)_{\mathcal{F}_{\text {slip }, h}^{n}} \\
& -\left(\boldsymbol{u}_{h}^{n} \cdot \boldsymbol{n}-\frac{\beta_{0}}{\Delta t} \boldsymbol{d}_{h}^{n} \cdot \boldsymbol{n}, \delta \boldsymbol{T}_{f}\left(\boldsymbol{v}_{h},-q_{h}\right) \boldsymbol{n} \cdot \boldsymbol{n}+(1-\delta) \boldsymbol{T}_{S}\left(\boldsymbol{w}_{h}\right) \boldsymbol{n} \cdot \boldsymbol{n}\right)_{\mathcal{F}_{\text {slip }, h}^{n}} \\
& +\left(\sigma_{\Sigma}\left(\boldsymbol{u}_{h}^{n} \cdot \boldsymbol{n}-\frac{\beta_{0}}{\Delta t} \boldsymbol{d}_{h}^{n} \cdot \boldsymbol{n}\right), \boldsymbol{v}_{h} \cdot \boldsymbol{n}-\boldsymbol{w}_{h} \cdot \boldsymbol{n}\right)_{\mathcal{F}_{\text {slip }, h}^{n}} ; \\
& A_{C, h}^{n}\left(\boldsymbol{u}_{h}^{n}, p_{h}^{n}, \boldsymbol{d}_{h}^{n} ; \boldsymbol{v}_{h}, q_{h}, \boldsymbol{w}_{h}\right)=-\left(\delta \boldsymbol{T}_{f}\left(\boldsymbol{u}_{h}^{n}, p_{h}^{n}\right) \boldsymbol{n} \cdot \boldsymbol{n}+(1-\delta) \boldsymbol{T}_{S}\left(\boldsymbol{d}_{h}^{n}\right) \boldsymbol{n} \cdot \boldsymbol{n}, \boldsymbol{v}_{h} \cdot \boldsymbol{n}-\boldsymbol{w}_{h} \cdot \boldsymbol{n}\right)_{\mathcal{F}_{C, h}^{n}} \\
& -\left(\boldsymbol{u}_{h}^{n} \cdot \boldsymbol{n}-\frac{\beta_{0}}{\Delta t} \boldsymbol{d}_{h}^{n} \cdot \boldsymbol{n}, \delta \boldsymbol{T}_{f}\left(\boldsymbol{v}_{h},-q_{h}\right) \boldsymbol{n} \cdot \boldsymbol{n}+(1-\delta) \boldsymbol{T}_{S}\left(\boldsymbol{w}_{h}\right) \boldsymbol{n} \cdot \boldsymbol{n}\right)_{\mathcal{F}_{C, h}^{n}} \\
& +\left(\sigma_{\Sigma}\left(\boldsymbol{u}_{h}^{n} \cdot \boldsymbol{n}-\frac{\beta_{0}}{\Delta t} \boldsymbol{d}_{h}^{n} \cdot \boldsymbol{n}\right), \boldsymbol{v}_{h} \cdot \boldsymbol{n}-\boldsymbol{w}_{h} \cdot \boldsymbol{n}\right)_{\mathcal{F}_{C, h}^{n}} \\
& -\left(\sigma_{C} g_{C}\left(\boldsymbol{d}_{h}^{n}\right), \boldsymbol{w}_{h} \cdot \boldsymbol{n}\right)_{\mathcal{F}_{C, h}^{n}} \\
& -\left(\delta\left(\boldsymbol{T}_{f}\left(\boldsymbol{u}_{h}^{n}, p_{h}^{n}\right) \boldsymbol{n}-\boldsymbol{T}_{S}\left(\boldsymbol{d}_{h}^{n}\right) \boldsymbol{n}\right) \cdot \boldsymbol{n}, \boldsymbol{w}_{h} \cdot \boldsymbol{n}\right)_{\mathcal{F}_{C, h}^{n}} \\
& -\left((1-\delta)\left(\boldsymbol{T}_{f}\left(\boldsymbol{u}_{h}^{n}, p_{h}^{n}\right) \boldsymbol{n}-\boldsymbol{T}_{S}\left(\boldsymbol{d}_{h}^{n}\right) \boldsymbol{n}\right) \cdot \boldsymbol{n}, \boldsymbol{v}_{h} \cdot \boldsymbol{n}\right)_{\mathcal{F}_{C, h}^{n}} ; \\
& F_{h}^{n}\left(\boldsymbol{v}_{h}, \boldsymbol{w}_{h}\right)=\rho_{f}\left(\widetilde{\boldsymbol{u}}_{h}^{n}, \boldsymbol{v}_{h}\right)_{\Omega_{f}^{n}}+\rho_{s}\left(\widetilde{\widetilde{\boldsymbol{d}}}_{h}^{n}, \widehat{\boldsymbol{w}}_{h}\right)_{\widehat{\Omega}_{s}} \\
& +\left(\widetilde{\boldsymbol{d}}_{h}{ }^{n}, \delta \boldsymbol{T}_{f}\left(\boldsymbol{v}_{h},-q_{h}\right) \boldsymbol{n}+(1-\delta) \boldsymbol{T}_{\mathcal{S}}\left(\boldsymbol{w}_{h}\right) \boldsymbol{n}\right)_{\mathcal{F}_{\text {no-slip }, h}^{n}} \\
& -\left(\sigma_{\Sigma} \widetilde{\boldsymbol{d}}_{h}{ }^{n}, \boldsymbol{v}_{h}-\boldsymbol{w}_{h}\right)_{\mathcal{F}_{\text {no-slip }, h}^{n}} \\
& +\left(\widetilde{\boldsymbol{d}}_{h}^{n} \cdot \boldsymbol{n}, \delta \boldsymbol{T}_{f}\left(\boldsymbol{v}_{h},-q_{h}\right) \boldsymbol{n} \cdot \boldsymbol{n}+(1-\delta) \boldsymbol{T}_{S}\left(\boldsymbol{w}_{h}\right) \boldsymbol{n} \cdot \boldsymbol{n}\right)_{\mathcal{F}_{\text {slip }, h}^{n} \cup \mathcal{F}_{C, h}^{n}} \\
& -\left(\sigma_{\Sigma} \widetilde{\boldsymbol{d}}_{h}^{n} \cdot \boldsymbol{n}, \boldsymbol{v}_{h} \cdot \boldsymbol{n}-\boldsymbol{w}_{h} \cdot \boldsymbol{n}\right)_{\mathcal{F}_{\text {slip }, h}^{n} \cup \mathcal{F}_{C, h}^{n}} \\
& +\left(\boldsymbol{f}_{f}, \boldsymbol{v}_{h}\right)_{\Omega_{f}^{n}}+\left(\widehat{f}_{s}, \widehat{\boldsymbol{w}}_{h}\right)_{\widehat{\Omega}_{s}}-\left(\sigma_{C} \mathcal{\varepsilon}, \boldsymbol{w}_{h} \cdot \boldsymbol{n}\right)_{\mathcal{F}_{C, h}^{n}} .
\end{aligned}
$$


In (3.11) the bilinear forms $a_{f}^{n}: V_{f, h}^{\ell, n} \times V_{f, h}^{\ell, n} \rightarrow \mathbb{R}, b^{n}: Q_{h}^{\ell, n} \times V_{f, h}^{\ell, n} \rightarrow \mathbb{R}$, the trilinear form $c^{n}: V_{f, h}^{\ell, n} \times V_{f, h}^{\ell, n} \times V_{f, h}^{\ell, n} \rightarrow \mathbb{R}$ and the Interior Penalty term for pressure stabilization $s_{h}^{n}: Q_{h}^{\ell, n} \times$ $Q_{h}^{\ell, n} \rightarrow \mathbb{R}$ are the ones given in Section 3.1 evaluated on the approximation of the fluid domain at time $t^{n}, \Omega_{f}^{n}$.

Remark 3.6. The domain $\Omega_{f}^{n}$ is computed as the set difference between the domain $\Omega$ and $\Omega_{s}^{n}$, i.e. $\Omega_{f}^{n}=\Omega \backslash \Omega_{s}^{n}$, where $\Omega_{s}^{n}$ is the solid domain in the current configuration obtained by applying the displacement $\widehat{\boldsymbol{d}}_{h}^{n}$ to $\widehat{\Omega}_{s}$ via the discrete Lagrangian map $\mathcal{L}_{h}^{n}: \widehat{\Omega}_{s} \rightarrow \Omega_{s}^{n}$ defined as $\mathcal{L}_{h}^{n}=\boldsymbol{I}_{\widehat{\Omega}_{s}}+\widehat{\boldsymbol{d}}_{h}^{n}$. The sets $\Sigma_{\text {no-slip }}^{n}, \Sigma_{\text {slip }}^{n}$ and $\Gamma_{C}^{n}$ are then obtained by restricting $\Omega_{f}^{n}$ to the corresponding interfaces.

We point out that in the fully discrete problem two sources of non-linearities are present: the convective term in the Navier-Stokes equations and the fact that the fluid domain $\Omega_{f}^{n}$ and the interfaces $\Sigma_{\text {no-slip, }}^{n} \Sigma_{\text {slip, }}^{n}, \Gamma_{C}^{n}$ are unknown.

To deal with all these non-linearities, we use a fixed point scheme. We denote by $k=0, \cdots, K^{\max }$, with $K^{\max } \geq 0$ chosen a priori, the index for the fixed point loop. At time $t^{n}$ and iteration $k>0$ :

a) the convection term in (3.11) is approximated by $c^{n}\left(\boldsymbol{u}_{h}^{n,(k-1)}, \boldsymbol{u}_{h}^{n,(k)}, \boldsymbol{v}_{h}\right)$;

b) the fluid domain and the interfaces in (3.11), (3.12), (3.13), (3.14), (3.15) are approximated by $\Omega_{f}^{n,(k-1)}, \Sigma_{\text {no-slip }}^{n,(k-1)}, \Sigma_{\text {slip }}^{n,(k-1)}$ and $\Gamma_{C}^{n,(k-1)}$, respectively.

The fixed point scheme stops when two consecutive structure displacements differ up to a prescribed tolerance, i.e. $\left\|\boldsymbol{d}_{h}^{n,(k)}-\boldsymbol{d}_{h}^{n,(k-1)}\right\|_{L^{2}}<t o l$, with tol chosen a priori, or the maximum number of iterations is reached, i.e. $k=K^{\max }$. In Algorithm 1, we detail the fixed point scheme. The choice of an exact (up to the tolerance) treatment of the nonlinearities has been mainly driven by the contact non-linearity, since an inexact treatment would provoke inaccuracies and possibly numerical instabilities.

The resulting linear system corresponding to the linearized FSI problem with contact arising at each fixed point iteration is solved by means of a direct method.

As initial guess $(k=0)$ of the fixed point scheme, we use extrapolations of order $r$ for $\boldsymbol{u}_{h}^{n,(0)}$ and $\boldsymbol{d}_{h}^{n,(0)}$ obtained by solutions at previous time steps.

\subsection{Extension to two deformable immersed structures}

In this section, we describe the main changes needed when extending the numerical formulation presented in Section 3.1 to the case of two immersed and deformable structures that may come into contact. In particular, $\Omega_{s}$ is the secondary body while $\Omega_{m}$ is the main one. In what follows, index $m$ refers to quantities in the main body. We denote by $\boldsymbol{n}_{\alpha}$ the normal unit vectors to $\Omega_{\alpha}$, for $\alpha=s, m$. 


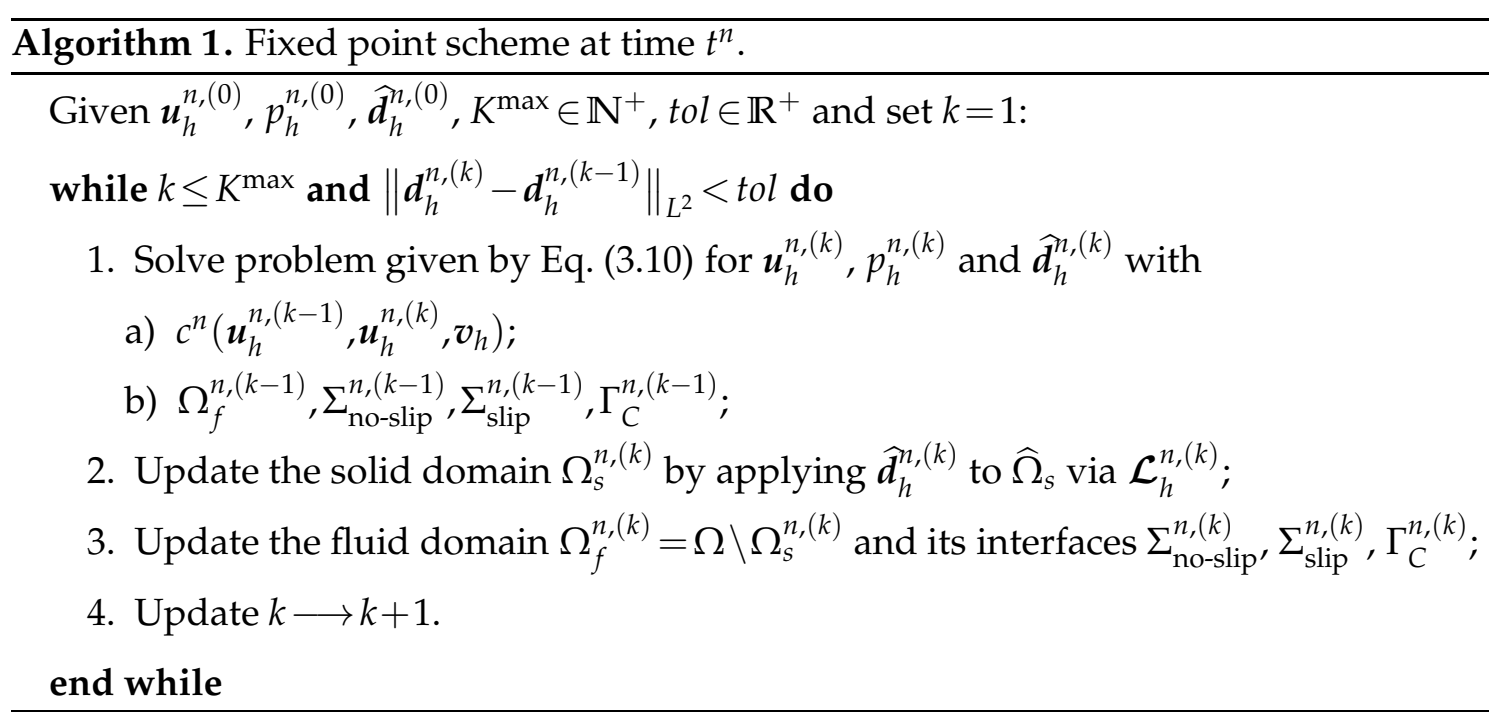

Referring to Fig. 3, the boundary of the solid domain $\partial \Omega_{\alpha}$ is partitioned into $\Sigma_{\text {no-slip }, \alpha}$ $\Sigma_{\text {slip, } \alpha}$ and $\Gamma_{C, \alpha}, \alpha=s, m$, as done in Section 2 . While for the secondary body these regions are defined as in Section 2, for the main one they are defined as the projection of the secondary regions onto the main boundary, i.e.

$$
\begin{aligned}
& \Gamma_{C, m}=\left\{\boldsymbol{x} \in \partial \Omega_{m}: \boldsymbol{x}=\Pi_{s \rightarrow m}(\boldsymbol{y}) \forall \boldsymbol{y} \in \Gamma_{C, s}\right\}, \\
& \Sigma_{\text {slip }, m}=\left\{\boldsymbol{x} \in \partial \Omega_{m}: \boldsymbol{x}=\Pi_{s \rightarrow m}(\boldsymbol{y}) \forall \boldsymbol{y} \in \Sigma_{\text {slip }, s}\right\}, \\
& \Sigma_{\text {no-slip }, m}=\partial \Omega_{m} \backslash\left(\Sigma_{\text {slip }, m} \cup \Gamma_{C, m}\right),
\end{aligned}
$$

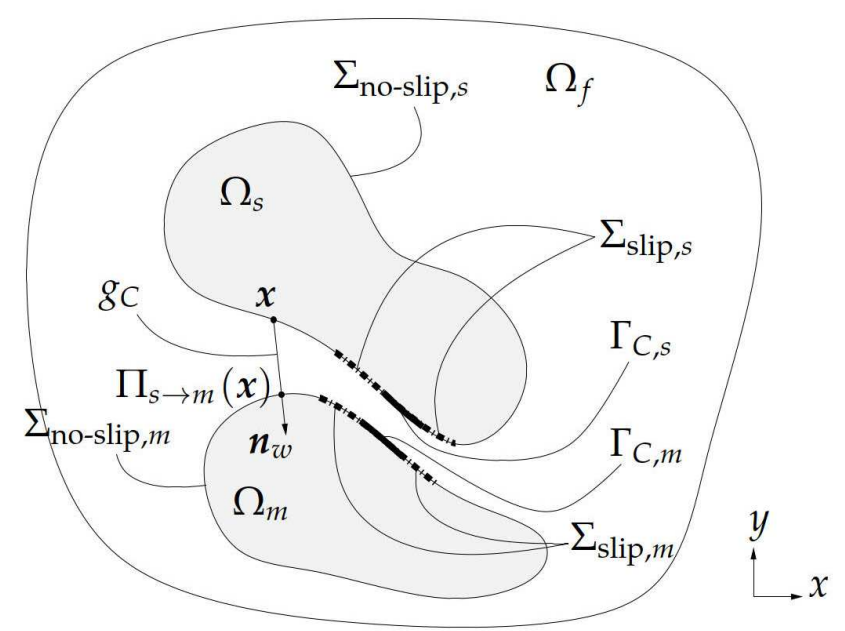

Figure 3: Contact between two deformable structures: sketch of the domains. $\partial \Omega_{\alpha}$ is partitioned in $\Sigma_{\text {no-slip, } \alpha}$ (solid line), $\Sigma_{\text {slip }, \alpha}$ (dash line) and $\Gamma_{C, \alpha}$ (bold line), $\alpha=s, m$. 
where $\Pi_{s \rightarrow m}: \partial \Omega_{s} \rightarrow \partial \Omega_{m}$ is the projection from the secondary domain to the main one defined as

$$
\Pi_{s \rightarrow m}(\boldsymbol{x})=\underset{\boldsymbol{y} \in \partial \Omega_{m}}{\operatorname{argmin}}\left|(\boldsymbol{x}-\boldsymbol{y}) \cdot \boldsymbol{n}_{m}(\boldsymbol{y})\right| \quad \forall \boldsymbol{x} \in \partial \Omega_{s} .
$$

The gap function $g_{C}$ is now defined as follows

$$
g_{C}(\boldsymbol{x})=\left(\Pi_{s \rightarrow m}(\boldsymbol{x})-\boldsymbol{x}\right) \cdot \boldsymbol{n}_{w}(\boldsymbol{x}),
$$

where $\boldsymbol{n}_{w}(\boldsymbol{x})=-\boldsymbol{n}_{m} \circ \prod_{s \rightarrow m}(\boldsymbol{x})$.

The main difference with respect to the formulation presented in Section 2 is the presence of the main body $\Omega_{m}$ that requires to introduce:

1. an elastodynamics equation for the body $\Omega_{m}$;

2. interface conditions on $\Sigma_{\text {no-slip, } \alpha}$ and $\Sigma_{\text {slip, } \alpha}, \alpha=s, m$, for both the structures, i.e. standard FSI conditions and (2.2), respectively;

3. a "contact force" on $\Gamma_{C, m}$ to balance the one acting on $\Gamma_{C, s}$. Accordingly, the balance of stresses on the contact regions $\Gamma_{C, S}$ and $\Gamma_{C, m}$ reads:

$$
\begin{array}{ll}
\boldsymbol{T}_{f}(\boldsymbol{u}, p) \boldsymbol{n} \cdot \boldsymbol{n}=\boldsymbol{T}_{S}\left(\boldsymbol{d}_{s}\right) \boldsymbol{n} \cdot \boldsymbol{n}-\lambda_{C} \quad \text { on } \Gamma_{C, S}, \\
\boldsymbol{T}_{s}\left(\boldsymbol{d}_{s}\right) \boldsymbol{n} \cdot \boldsymbol{\tau}-\varphi_{C}=0 \quad \text { on } \Gamma_{C, S}, \\
\boldsymbol{T}_{f}(\boldsymbol{u}, p) \boldsymbol{n} \cdot \boldsymbol{n}=\boldsymbol{T}_{m}\left(\boldsymbol{d}_{m}\right) \boldsymbol{n} \cdot \boldsymbol{n}+\lambda_{C} \quad \text { on } \Gamma_{C, m}, \\
\boldsymbol{T}_{m}\left(\boldsymbol{d}_{m}\right) \boldsymbol{n} \cdot \boldsymbol{\tau}+\varphi_{C}=0 \quad \text { on } \Gamma_{C, m} .
\end{array}
$$

The corresponding PolyDG formulations are derived as done in Sections 3.1 and 3.2, where all the terms involving $\Omega_{s}$ and its interfaces are duplicated for $\Omega_{m}$. We point out that, according to a main/secondary approach, the contact conditions (2.3o)-(2.3p) are applied only on the secondary body $\Omega_{s}$. Accordingly, in the form $A_{C, h}$ corresponding to the contact regions, the contact penalty term reads

$$
-\left(\sigma_{C} g_{C}\left(\boldsymbol{d}_{s, h}, \boldsymbol{d}_{m, h}\right), \boldsymbol{w}_{s, h} \cdot \boldsymbol{n}-\boldsymbol{w}_{m, h} \cdot \boldsymbol{n}\right)_{\mathcal{F}_{C, s, h}}
$$

where $\mathcal{F}_{C, s, h}$ is the set of edges of $\Gamma_{C, s}$ and again we have highlighted the dependence of $g_{C}$ on the structure displacements.

\subsection{Implementation details}

One of the most difficult tasks for the methods employing elements of arbitrary shape is the construction of the quadrature rules over the polygonal elements. Often, quadrature is carried out based on employing sub-tessellation: each polygon is divided into triangle/quads where classical Gaussian rules are employed. Recently, efficient quadrature formulae that do not require the decomposition of the polygonal element into simplices 


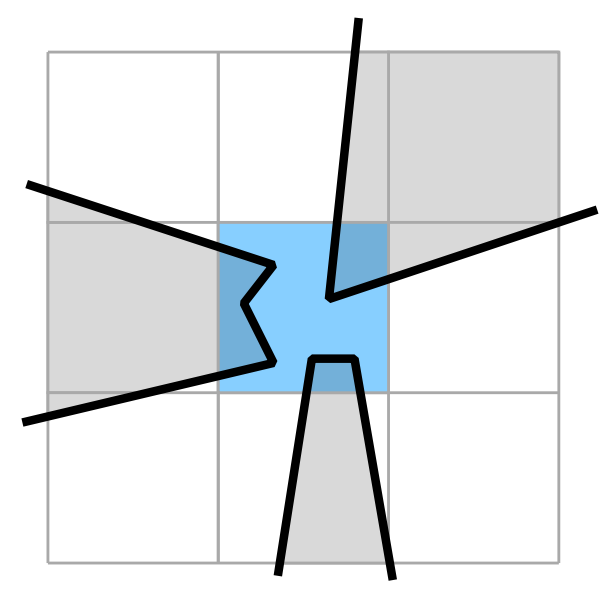

Figure 4: A possible general intersection between an element (blue) of the background mesh (white) and three structure meshes (gray).

have been proposed in [9] for the efficient computation of integrals involving polynomial functions. This technique does not require the definition of quadrature nodes and weights, often referred as "quadrature-free" method, but only the evaluation of the integrand polynomial together with its derivatives on the vertices of the polygon thanks to a recursive application of the Stokes' theorem.

Another important aspect is the robustness of the proposed method with respect to the shape of the polygonal elements. As shown in the numerical tests in [11], the PolyDG method seems to be robust even in presence of very small elements and with respect to possible anisotropy. We point out that the polygonal elements generated by the intersection of the fluid and structure meshes, see e.g. Fig. 2 (bottom-right), might lead to pathological configurations: each element $K$ of the background mesh $\mathcal{T}_{h}$ can be intersected by an arbitrary number of structure meshes, each face of $K$ can be crossed an arbitrary number of times, sharp corners within an element are allowed and the resulting polygonal element can be non-convex, see Fig. 4 for an example of a possible configuration. The proposed PolyDG method seems to be robust also in these cases.

A special issue concerning the methods involving a moving interface that requires to update the discrete space at each time step is the computation of the integrals involving function defined on different computational domains. For instance, the term

$$
\rho_{f}\left(\widetilde{\boldsymbol{u}}_{h}^{n}, \boldsymbol{v}_{h}\right)_{\Omega_{f}^{n}}
$$

appearing in Eq. (3.15), requires the evaluation of $\boldsymbol{v}_{h}$, defined in $V_{f, h}^{\ell, n}$, and of $\widetilde{\boldsymbol{u}}_{h}^{n}$, which is a combination of functions defined in $V_{f, h}^{\ell, n-i}$, for $i=1, \cdots, r$. For this reason, in order to numerically evaluate the integral, it is necessary to project the function $\widetilde{\boldsymbol{u}}_{h}^{n}$ on $V_{f, h}^{\ell, n}$. In what follows, for the sake of exposition, we consider $r=1$, i.e. the implicit Euler time scheme, so that we have $\rho_{f}\left(\boldsymbol{u}_{h}^{n-1}, \boldsymbol{v}_{h}\right)_{\Omega_{f}^{n}}$, and we define $\Pi_{n}^{n-1} \boldsymbol{u}_{h}^{n-1} \in \boldsymbol{V}_{f, h}^{\ell, n}$ the extension of 

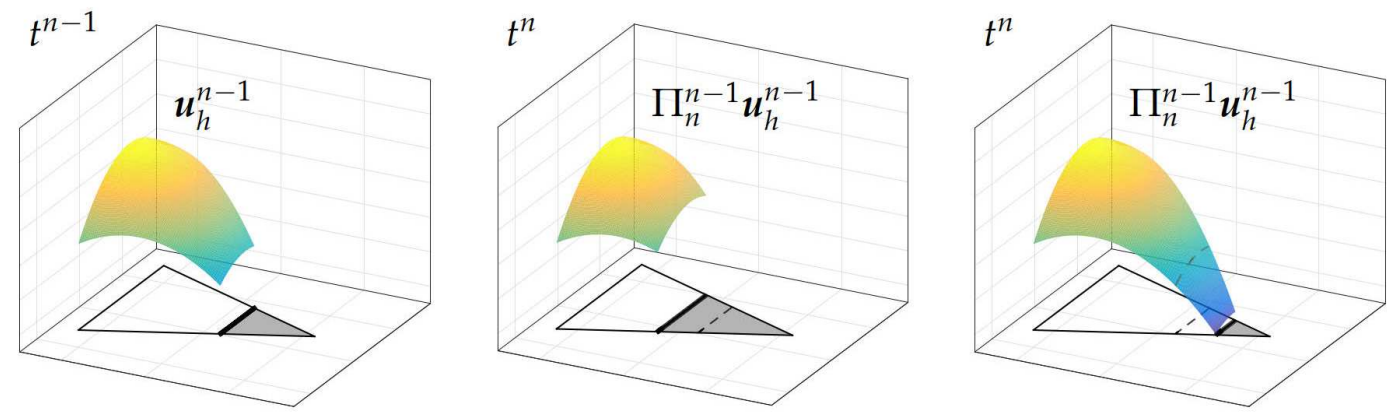

Figure 5: Generation of $\Pi_{n}^{n-1} \boldsymbol{u}_{h}^{n-1}$ on an element $K$ (white). The shaded region represents the portion of the original background element covered by the structure, while the bold and the dashed lines are the current and previous positions of the interface $\Sigma$, respectively. Left: original solution $\boldsymbol{u}_{h}^{n-1}$ defined on $\boldsymbol{V}_{f, h}^{\ell, n-1}$. Center: solution $\Pi_{n}^{n-1} \boldsymbol{u}_{h}^{n-1}$ restricted on the new (shortened) element $K\left(t^{n}\right)$. Right: solution $\Pi_{n}^{n-1} \boldsymbol{u}^{n-1}$ extended to the new (elongated) element $K\left(t^{n}\right)$.

$\boldsymbol{u}_{h}^{n-1}$ on $\boldsymbol{V}_{f, h}^{\ell, n}$, where $\Pi_{n}^{n-1}: \boldsymbol{V}_{f, h}^{\ell, n-1} \rightarrow \boldsymbol{V}_{f, h}^{\ell, n}$ is the $L^{2}$ projection operator. For each element $K$ in the computational domain associated with the domain $V_{f, h}^{\ell, n}$, the $L^{2}$ projection operator $\Pi_{n}^{n-1}$ is defined as follows:

- if the element $K$ remains the same from time $t^{n-1}$ to $t^{n}$, the extension operator is the identity operator, i.e. $\left.\Pi_{n}^{n-1} \boldsymbol{u}_{h}^{n-1}\right|_{K}=\left.\boldsymbol{u}_{h}^{n-1}\right|_{K}$;

- if the element $K$ shortens from time $t^{n-1}$ to $t^{n}$ due to the moving of the interface $\Sigma$, see Fig. 5 (left and center), the extension operator is actually a restriction of $\boldsymbol{u}_{h}^{n-1}$ on the new element $K$ at time $t^{n}$, i.e. $\left.\Pi_{n}^{n-1} \boldsymbol{u}_{h}^{n-1}\right|_{K\left(t^{n}\right)}=\left.\boldsymbol{u}_{h}^{n-1}\right|_{K\left(t^{n}\right)}$;

- if the element $K$ elongates from time $t^{n-1}$ to $t^{n}$ due to the moving of the interface $\Sigma$, see Fig. 5 (left and right), the extension operator computes an extrapolation of order $\ell$ of the function $\boldsymbol{u}_{h}^{n-1}$ on the new element $K$ at time $t^{n}$.

After the generation of $\Pi_{n}^{n-1} \boldsymbol{u}_{h}^{n-1}$, the actual integral to compute is

$$
\rho_{f}\left(\Pi_{n}^{n-1} \boldsymbol{u}_{h}^{n-1}, \boldsymbol{v}_{h}\right)_{\Omega_{f}^{n}} .
$$

Similar approaches have already been proposed in $[4,88]$.

\section{Numerical examples}

We present here two numerical experiments in two-dimensions: in the first one (test I), cf. Section 4.1, we study the dynamics of a falling elastic ball immersed in a fluid and bouncing on the ground. With this test case, we aim at investigating the sensitivity 
on the contact parameters introduced in the numerical formulation and at showing the reliability of the method for a high order discretization. In the second example (test II), cf. Section 4.2, we consider a more complex geometry and at high Reynolds regime. In particular, we consider the case of two immersed structures that come into contact, see Section 3.3.

For all the numerical tests, we set $r=1, \delta=1$ and $\gamma_{f}=\gamma_{s}=\gamma_{\Sigma}=10$. The tolerance for the stopping criterion in the fixed point scheme is set to $t o l=10^{-6}$.

\subsection{Ball falling to the ground}

In this example we consider an elastic ball $\Omega_{s}$ of radius $0.05 \mathrm{~cm}$, initially centered at $(0.25,0.175)$, falling in the air towards the rigid ground $\Gamma_{w}=\{(x, y): y=0\}$ due to the acceleration $g=(0,-980.655) \mathrm{cm} / \mathrm{s}^{2}$. The size of the fluid domain $\Omega_{f}$ is $0.5 \mathrm{~cm} \times 0.325 \mathrm{~cm}$ with the bottom-left corner placed in the origin $O=(0,0)$. The bottom boundary of the fluid domain corresponds to the rigid ground $\Gamma_{w}$, see Fig. 6 (left). The physical parameters for the fluid and solid are summarized in Table 1.

Table 1: Physical parameters for the fluid and the structure for the two test cases.

\begin{tabular}{||lccc||}
\hline Fluid parameters & & Test I & Test II \\
\hline$\rho_{f}\left(\mathrm{~g} / \mathrm{cm}^{2}\right)$ & density & $1.2 \cdot 10^{-3}$ & 1.0 \\
$\mu_{f}(\mathrm{~g} / \mathrm{s})$ & viscosity & $0.2 \cdot 10^{-3}$ & 0.035 \\
\hline Structure parameters & & & \\
\hline$\rho_{S}\left(\mathrm{~g} / \mathrm{cm}^{2}\right)$ & density & 0.1 & 1.2 \\
$E(\mathrm{dyne} / \mathrm{cm})$ & Young's module & $10^{3}$ & $4 \cdot 10^{6}$ \\
$v$ & Poisson's ratio & 0.45 & 0.45 \\
\hline
\end{tabular}

The air and the ball are initially at rest. On the upper boundary of the fluid domain we impose a homogeneous Neumann condition, while on the left, right and lower boundaries we set $\boldsymbol{u}=\mathbf{0}$. We consider a final time $T=0.03 \mathrm{~s}$.

The fluid and structure meshes are composed of about $1.6 \cdot 10^{3}$ and 80 elements, respectively, with a characteristic mesh size $h=1.6 \cdot 10^{-2} \mathrm{~cm}$, see Fig. 6 (right). The contact parameters are set to $\gamma_{C}=1, \varepsilon=0.1 \mathrm{~h}=1.6 \cdot 10^{-3} \mathrm{~cm}, g_{\text {slip }}=1.5 \mathrm{~h}=2.4 \cdot 10^{-2} \mathrm{~cm}$ and we consider a time step $\Delta t=10^{-4} \mathrm{~s}$. Regarding the fixed point scheme, we set a maximum number of iterations $K^{\max }=20$. Next, we present a series of tests to demonstrate the robustness of our scheme with respect to the contact parameters $\gamma_{C}, \varepsilon$ and $g_{\text {slip }}$, and to the discretization parameter $\Delta t$, by comparing the $y$-position of the ball at the point $A$. For these tests, we set $\ell=2$.

In Fig. 7 (top-left), we report the results obtained by considering $\gamma_{C}=$ $\{0.1,0.6,0.8,1,2,4\}$. We notice that by increasing the penalty parameter the bouncing of the ball is reduced, yielding the ball to remain in contact with the ground for the largest value of the parameter, namely $\gamma_{C}=4$. In Fig. 7 (top-right), we show the results obtained 

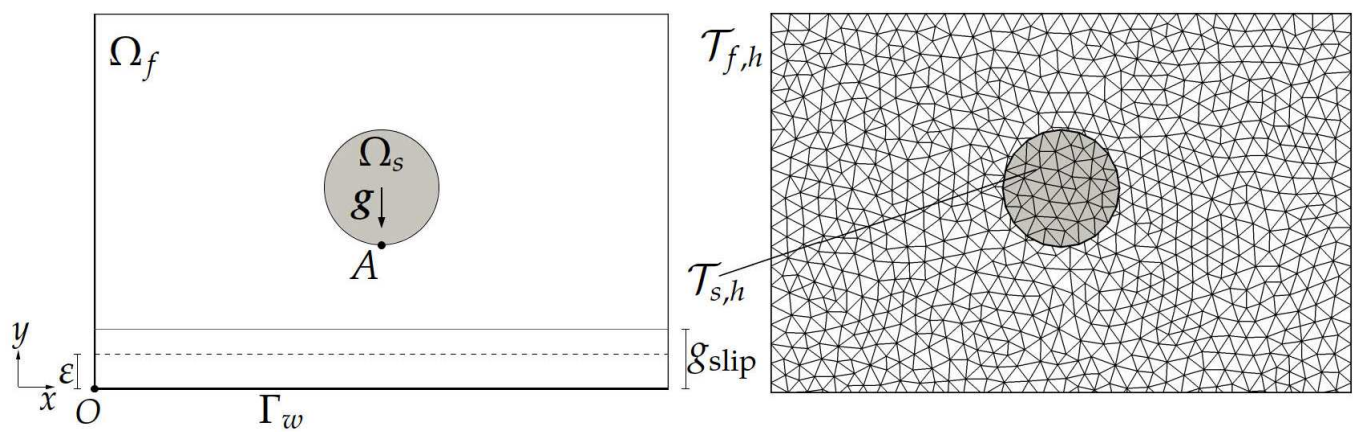

Figure 6: Left: sketch of the domains for the falling ball test case (test I). Right: the fluid and structure meshes $\mathcal{T}_{f, h}$ and $\mathcal{T}_{s, h}$, respectively.
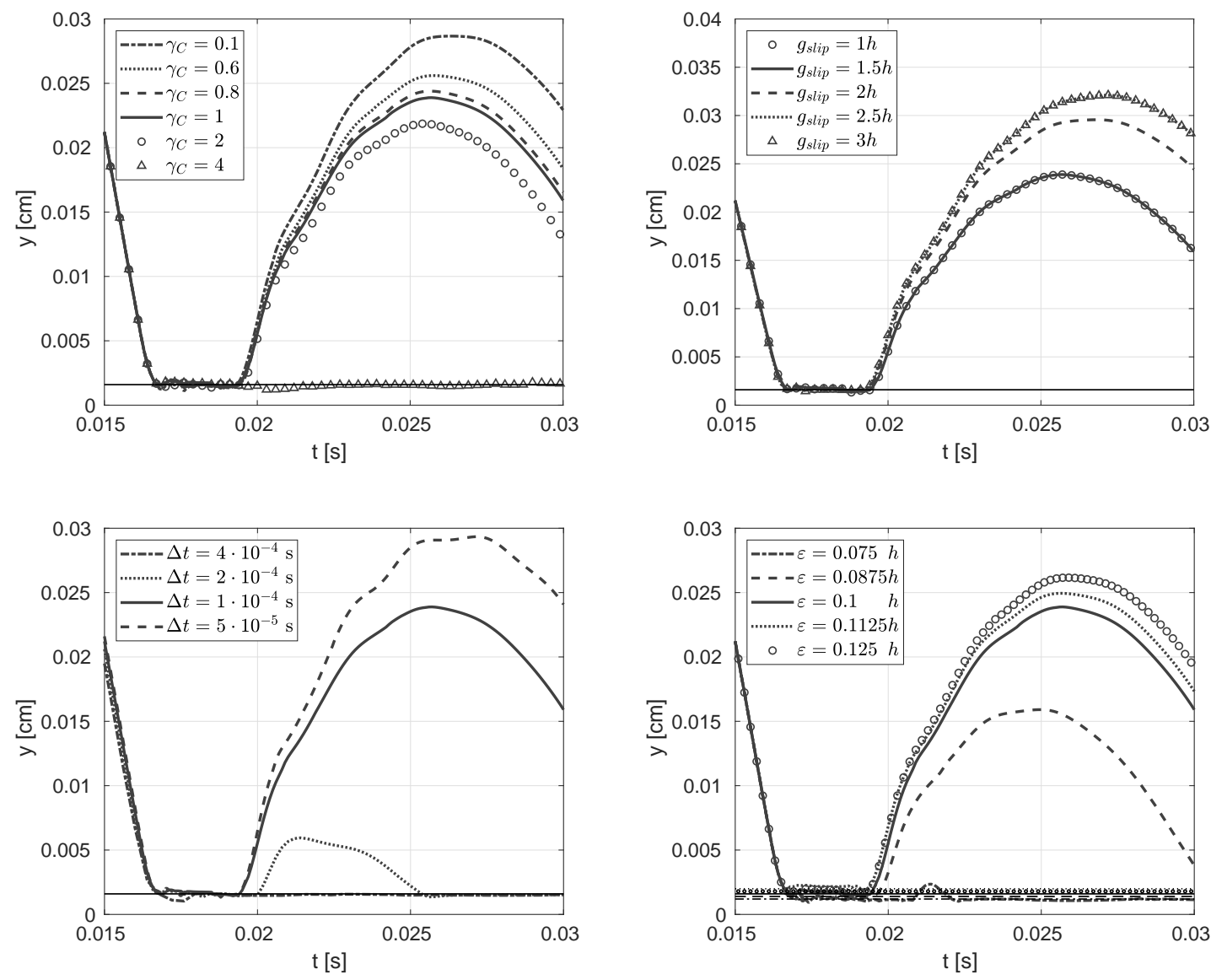

Figure 7: $y$-position of the ball in the point $A$ as a function of time $t$ by varying $\gamma_{C}$ (top-left), $g_{\text {slip }}$ (top-right),

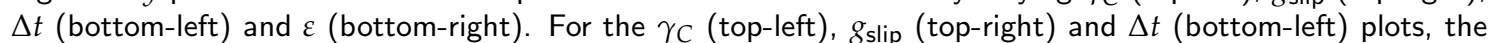
continuous horizontal line denotes the distance $\varepsilon=0.1 \mathrm{~h}$ to the ground. For the case of varying $\varepsilon$ (bottom-right), the horizontal lines denote the different distances $\varepsilon$ to the ground according to the legend (test I). 


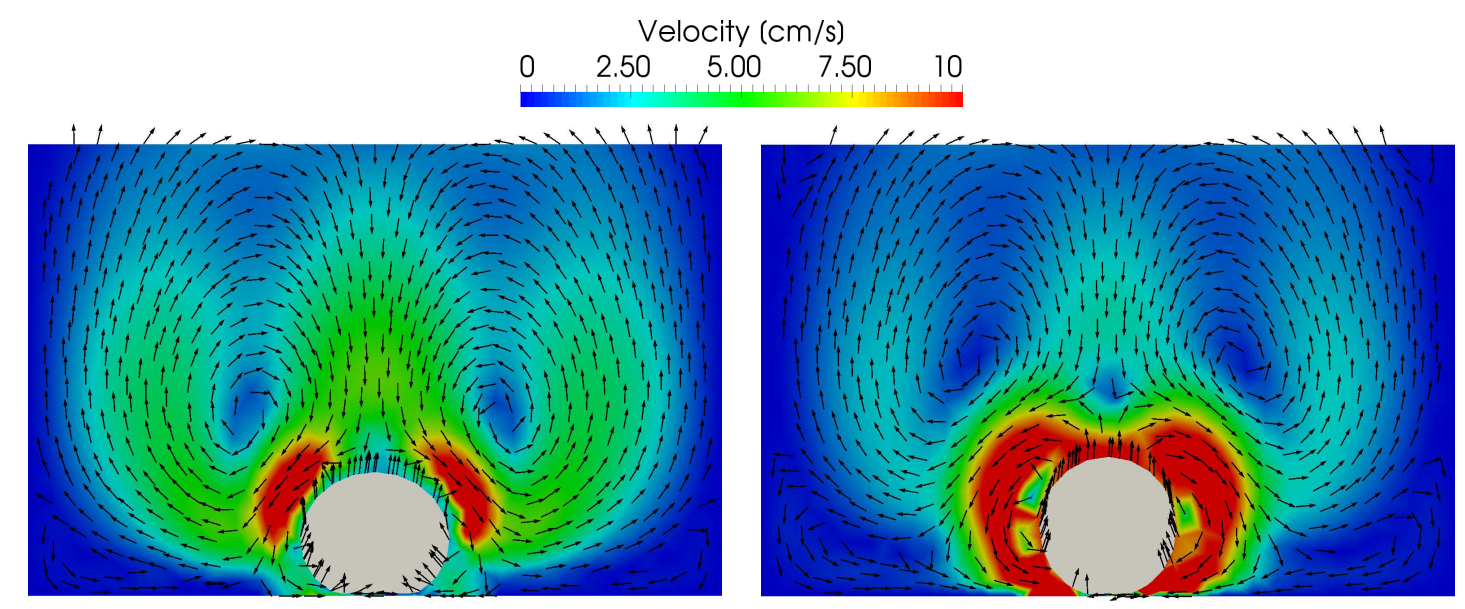

Figure 8: Velocity field and position of the ball at time $t=0.0179 \mathrm{~s}$ (left) and $t=0.0190 \mathrm{~s}$ (right) (test I).

by changing the threshold distance $g_{\text {slip }}=\{1,1.5,2,2.5,3\} \mathrm{h}$. As the value of $g_{\text {slip }}$ increases, we notice as expected that the height reached by the ball after bouncing increases. In Fig. 7 (bottom-left), we show the dependence of the dynamics of the ball on the time discretization, taking $\Delta t=\{0.5,1,2,4\} \cdot 10^{-4} \mathrm{~s}$. We notice that if the time step is not small enough, e.g. $\Delta t \geq 2 \cdot 10^{-4} \mathrm{~s}$, the numerical method is not able to properly detect the contact by hampering the bounce of the ball, for $\Delta t=2 \cdot 10^{-4} \mathrm{~s}$, or completely missing the releasing phase, for $\Delta t=4 \cdot 10^{-4} \mathrm{~s}$, and leading to a wrong dynamics. As soon as the time step is reduced the bouncing of the ball becomes more evident and the method is able to capture the high frequency oscillations of the ball. In Fig. 7 (bottom-right), we plot the results for different values of $\varepsilon=\{0.075,0.0875,0.1,0.1125,0.125\} h$. During the contact phase, we notice that, for increasing values of $\varepsilon$, as expected the position of the ball at point $A$ increases, though it may go below the prescribed threshold distance $\varepsilon$. For the smallest value, i.e. $\varepsilon=0.075 \mathrm{~h}$, the method is not able to reproduce the bounce of the ball, while for $\varepsilon \geq 0.1 \mathrm{~h}$ the results are similar.

In Fig. 8 , we plot the velocity field and the position of the ball at time $t=0.0179 \mathrm{~s}$ where it exhibits the maximum compression on the ground (left), and at time $t=0.0190$ s where the ball starts to move away from the ground (right).

By considering the following parameters, $\Delta t=10^{-4} \mathrm{~s}, \gamma_{C}=1, \varepsilon=0.1 \mathrm{~h}=1.6 \cdot 10^{-3} \mathrm{~cm}$, $g_{\text {slip }}=1.5 \mathrm{~h}=2.4 \cdot 10^{-2} \mathrm{~cm}$ and $K^{\max }=20$, we plot in Fig. 9 the number of iterations of the fixed point scheme at each time (left) and the corresponding residual defined as $\left\|\boldsymbol{d}_{h}^{n,(k)}-\boldsymbol{d}_{h}^{n,(k-1)}\right\|_{L^{2}}$, see Algorithm 1. We notice that outside the contact phase, the fixed point scheme requires about 5 iterations to converge, while during the contact, i.e. $t \in[0.015,0.02], 20$ iterations may be not enough to achieve convergence, though the residual is of order $2 \cdot 10^{-3}$.

In Fig. 10, we show a comparison of the $y$-displacement of the ball in the point $A$ for different choices of the polynomial degree, i.e. $\ell=1,2,3$. We notice that the falling and the 

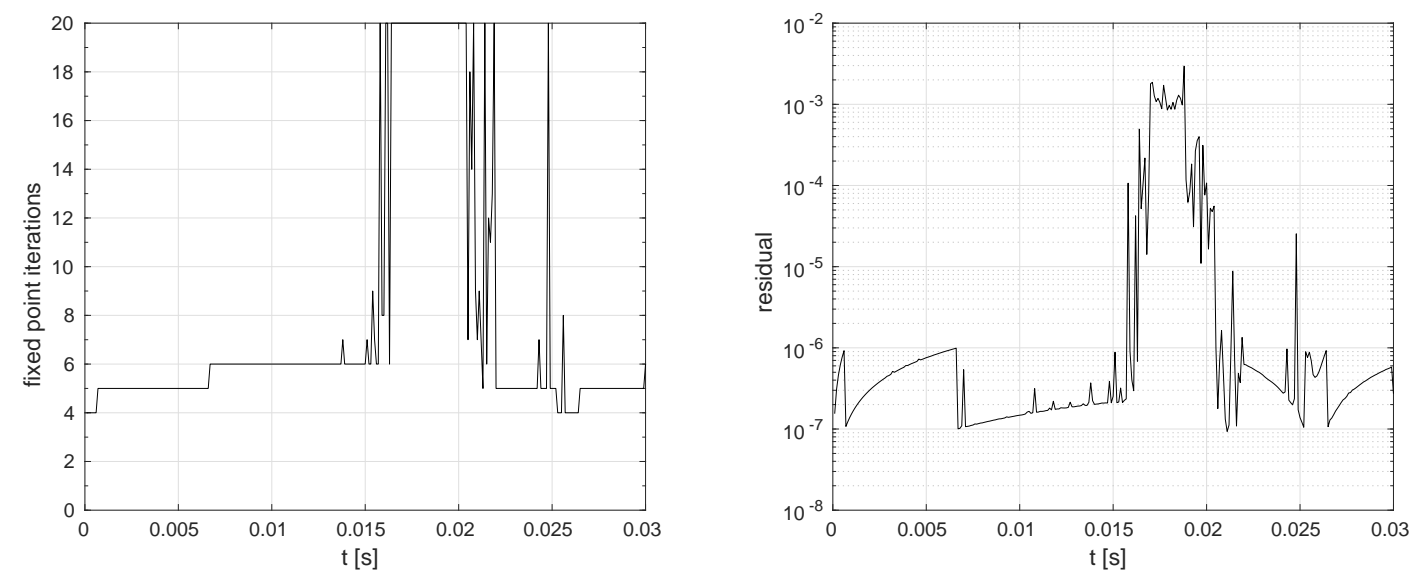

Figure 9: Number of iterations of the fixed point scheme (left) and residual (right) at each time step (test I).
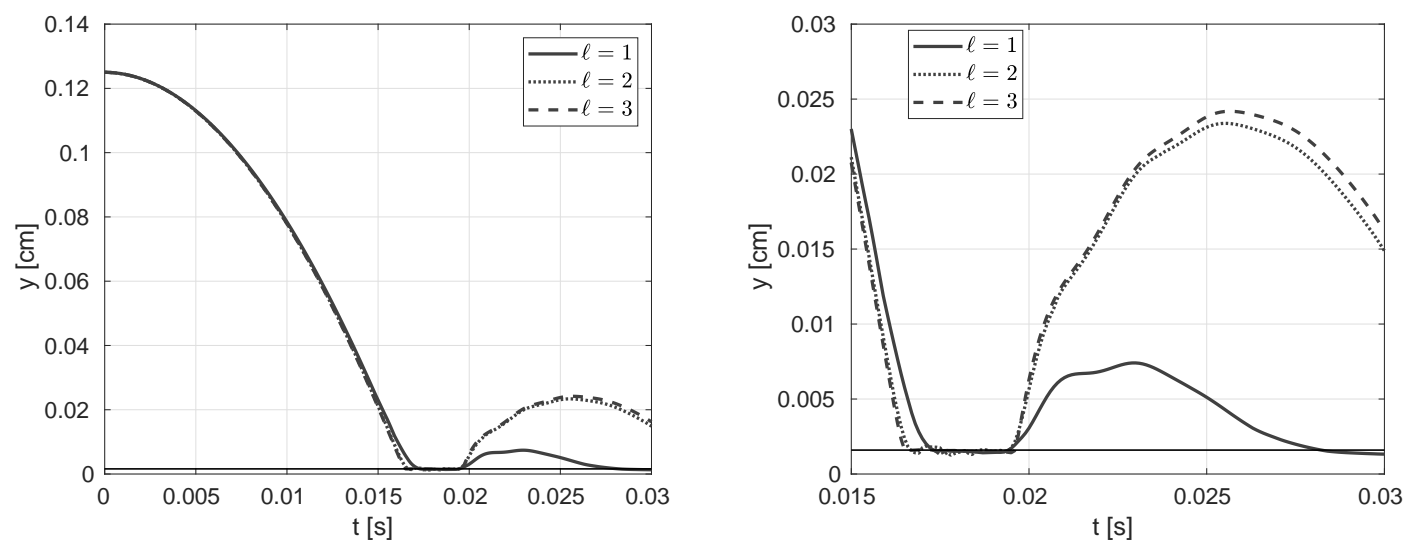

Figure 10: $y$-position of the ball in the point $A$ as a function of time $t$ for $\ell=1$ (continuous line), $\ell=2$ (dotted line) and $\ell=3$ (dashed line). The continuous horizontal line denotes the distance $\varepsilon=0.1 \mathrm{~h}$ to the ground. Left: evolution on the entire simulation. Right: detail at the contact and bouncing phases (test I).

contact phases of the ball are very similar, see Fig. 10 (left). However, the bouncing phase of the ball is very different, see Fig. 10 (right): compared to the case $\ell=1$, for $\ell=2$ and $\ell=3$ the ball reaches a higher (and comparable) height. This phenomenon is not reproduced in the case $\ell=1$ probably due to the dissipative error and the stiffness characterizing a low accuracy in space; on the other hand, the cases $\ell=2$ and $\ell=3$ are very similar.

Finally, we set the polynomial degree $\ell=4$ and we carry out a numerical test to show the capability of the method to perform simulations at high-order degree. We consider the same physical and numerical parameters as in the previous experiment but a smaller fluid domain to limit the computational cost. In Fig. 11, we plot the numerical velocity and the position of the ball at time $t=0.0071 \mathrm{~s}$ (left) and the evolution in time of the $y$-position of the ball at the point $A$ (right). 

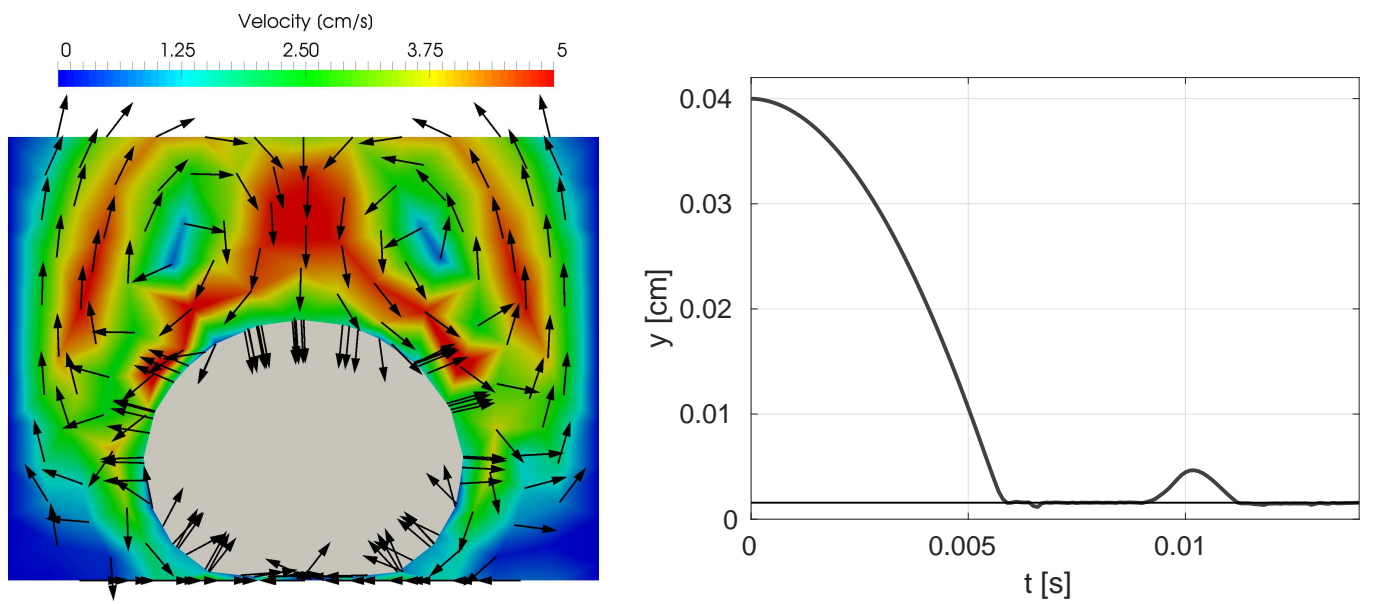

Figure 11: Case $\ell=4$. Left: Velocity field and position of the ball at time $t=0.0071 \mathrm{~s}$. Right: $y$-position of the ball in the point $A$ as a function of time $t$. The continuous horizontal line denotes the distance $\varepsilon=0.1 \mathrm{~h}$ to the ground (test I).

This test showed that the proposed method is able to simulate the contact between an immersed structure with a rigid boundary in a fluid-structure interaction context, possibly also with a high order method. However, we notice the importance to properly choose the values of the numerical parameters. In particular, as expected the time step $\Delta t$ has to be small enough to capture the physics of the problem. Moreover, as expected the contact parameters $\gamma_{C}, g_{\text {slip }}$ and $\varepsilon$ may significantly affect the dynamics of the system. For this test, we notice that a good choice for these parameters seems to be:

- $\gamma_{C} \in[0.6,1]$; for smaller and greater values, the bouncing is enhanced and hampered, respectively, and for the largest value, i.e. $\gamma_{C}=4$, the ball never releases from the ground by indicating that the penalty parameter is too large;

- $g_{\text {slip }} \in[1.5 h, 2.5 h]$; for smaller and greater values the solution does not change significantly, meaning that the parameter has no influence outside the range;

- $\Delta t \leq 10^{-4} s$ to capture the correct dynamics and the high frequency oscillations of the structure;

- $\varepsilon \geq 0.1 \mathrm{~h}$; for smaller values the dynamics changes significantly yielding a too low bounce of the ball.

\subsection{Dynamics in an idealized immersed valve}

Referring to the formulation and notation described in Section 3.3, we consider the idealized axisymmetric 2D valve shown in Fig. 12 (left) with two immersed leaflets. The geometry of the fluid domain $\Omega_{f}$ and the solid ones, $\Omega_{s}$ and $\Omega_{m}$, are defined according to $[56]$. 

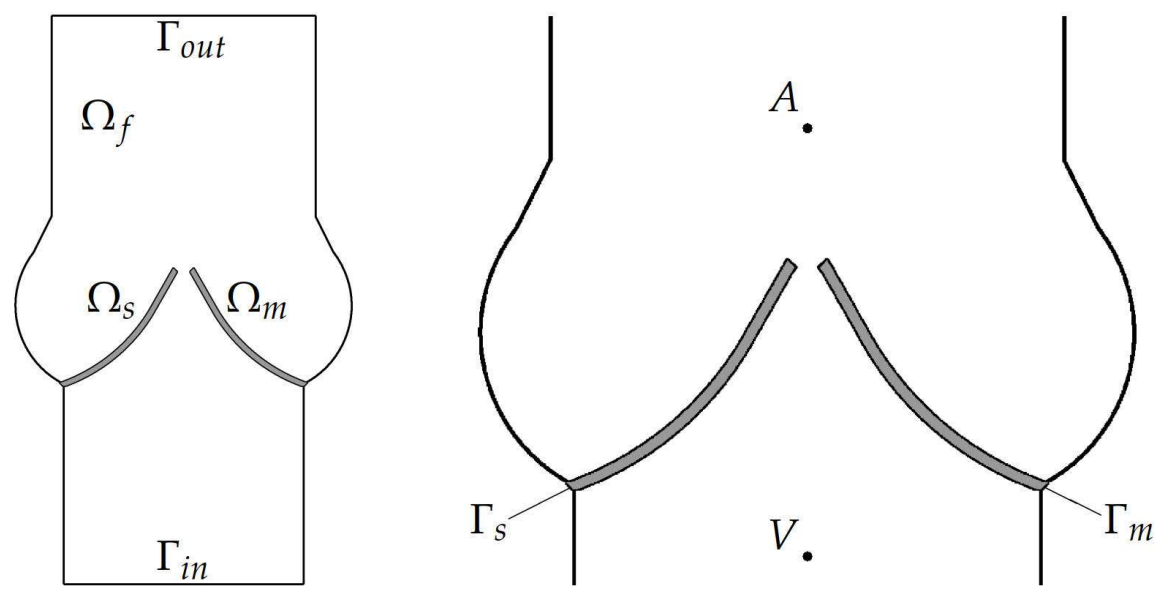

Figure 12: Left: sketch of the domain for the valve test case. Right: detail of the domain close to the two immersed structures. The points $A$ and $V$ are the probes location for measuring the pressure (test II).

The material properties in this case are taken from the hemodynamic regime, see Table 1 . The system is initially at rest, and the dynamics is driven by an idealized timedependent pressure profile at the inlet $\Gamma_{\text {in }}$, see Fig. 13 (top-left). At the outlet $\Gamma_{\text {out }}$ we impose $\boldsymbol{T}_{f} \boldsymbol{n}=\mathbf{0}$, while on the remaining fluid boundary we impose $\boldsymbol{u}=\mathbf{0}$. On the structures boundaries $\Gamma_{s}$ and $\Gamma_{m}$, we impose $\boldsymbol{d}_{s}=\boldsymbol{d}_{m}=\mathbf{0}$, see Fig. 12 (right). We consider $T=0.8 \mathrm{~s}$.

For the numerical simulation, we consider $\ell=2, \Delta t=0.0025 \mathrm{~s}, K^{\max }=5$, the fluid mesh size $h_{f}=0.13 \mathrm{~cm}$ that corresponds to $2.1 \cdot 10^{3}$ elements, the solid mesh size $h_{s}=h_{m}=0.02 \mathrm{~cm}$ that corresponds to $3 \cdot 10^{2}$ elements, $\varepsilon=0.1 h, \gamma_{C}=6 \cdot 10^{-4}$ and $g_{\text {slip }}=1.5 h$. To prevent instabilities at the outlet due to the backflow, we prescribe a backflow stabilization term, see e.g. [70], at $\Gamma_{\text {out }}$.

In Fig. 13, top-left, we plot the evolution of the pressure in time in the points $V$ and $A$ reported in Fig. 12, right, while in Fig. 13, top-right, we report the distance between the leaflets, representing the aperture of the valve, computed as the minimum value over $x \in \partial \Omega_{S}$ of the gap function $g_{C}(\boldsymbol{x})$. A zoom during the contact phase is shown in Fig. 13, bottom-left and bottom-right, for the pressure and the minimum distance, respectively. In Fig. 14, we report the velocity and pressure fields of the fluid at time $t=0.375 \mathrm{~s}$ (top) and $t=0.675 \mathrm{~s}$ (bottom). We see the complex fluid-dynamics developed in the valve and the configuration of the valve during the closing phase.

In Fig. 13 (top-left), we notice that during systole, i.e. for $0 \leq t \leq 0.2$, the ventricular pressure is higher than the aortic one and hence the leaflets open. For time $0.2 \leq t \leq 0.4$, the pressure at the inlet drops to zero and the blood flow starts to decelerate. As time tends to $0.4 \mathrm{~s}$, the difference between the ventricular and aortic pressure decreases. Notice that, downstream of the leaflets, two vortices appear and propagate towards the outlet $\Gamma_{\text {out }}$, see Fig. 14 (top), causing an oscillation of the tips of the leaflets.

In Fig. 13 (top-left), during diastole, i.e. for $0.4 \leq t \leq 0.6$, the pressure difference be- 

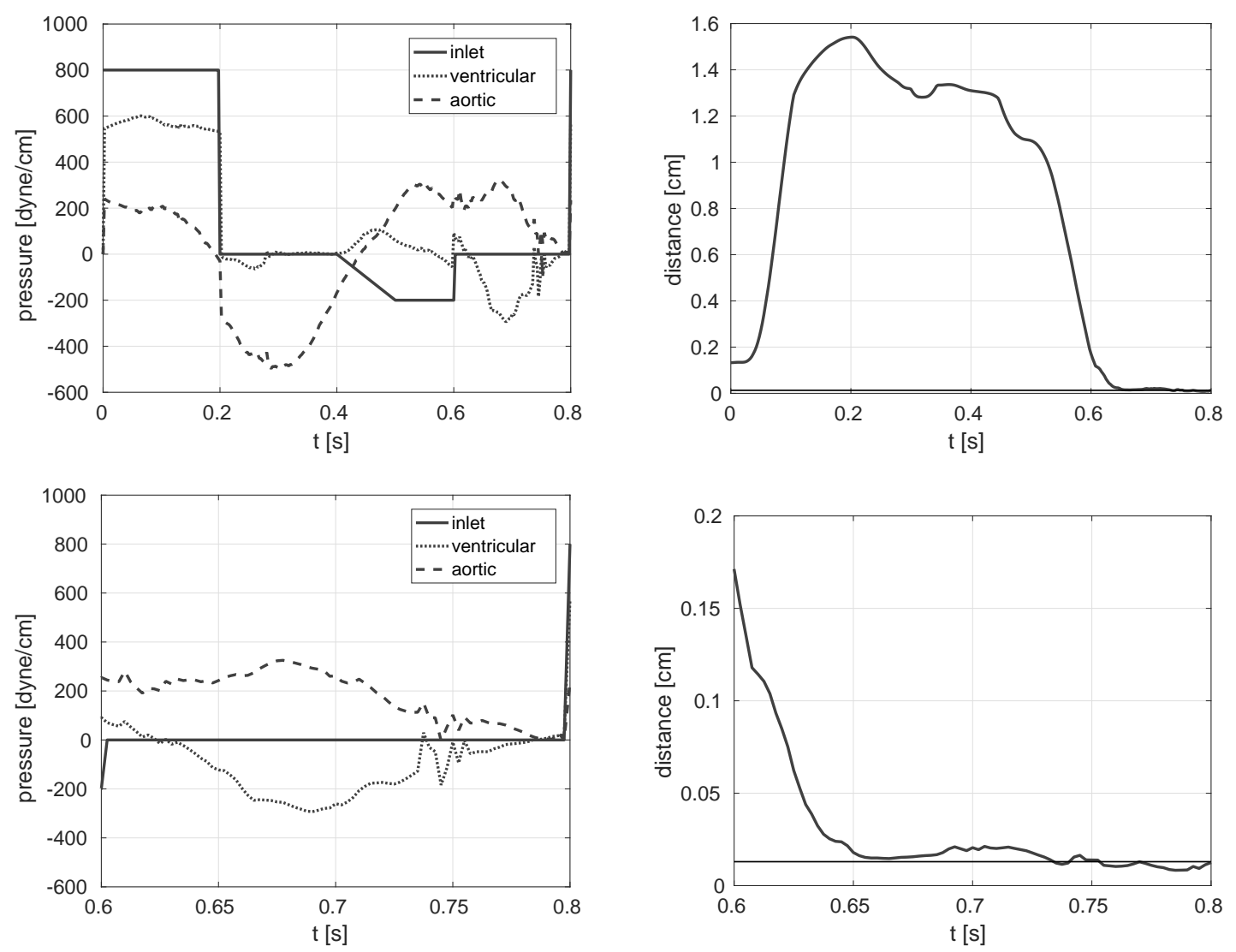

Figure 13: Left: plot of the pressure profile over time prescribed at the inlet $\Gamma_{\text {in }}$ and computed at points $V$ (ventricle) and $A$ (aorta) for the full simulation (top) and the detail during the contact phase (bottom). Right: plot of the minimum distance between the leaflets for the full simulation (top) and the detail during the contact phase (bottom). The continuous horizontal line denotes the distance $\varepsilon=0.1 \mathrm{~h}$ between the leaflets (test II).

comes zero for $t \simeq 0.5 \mathrm{~s}$, and then the aortic pressure becomes higher than the ventricular one. As a consequence, the leaflets start to move closer. Finally, in the time interval $0.6 \leq t \leq 0.8$, the leaflets come into contact, see Fig. 14 (bottom), and as time approaches $0.8 \mathrm{~s}$ the ventricular and aortic pressures return to zero, allowing the leaflets to return to the initial configuration. In Fig. 13 (bottom-left), we plot a zoom of the pressure during the contact phase: the ventricular and aortic pressures present some oscillations due to a local numerical instability arising near the contact region. In Fig. 13 (bottom-right), we plot the behaviour of the minimum distance during the contact phase, showing that it remains near the threshold distance $\varepsilon$. The maximum velocity reached by the fluid is about $41 \mathrm{~cm} / \mathrm{s}$ corresponding to a Reynolds number $R e=1600$. Notice that, though we have employed an idealized inlet profile and a simple linear elastic model for the leaflets, the method is able to reproduce the basic opening and closing mechanism of a valve, see e.g. $[56,81]$. 

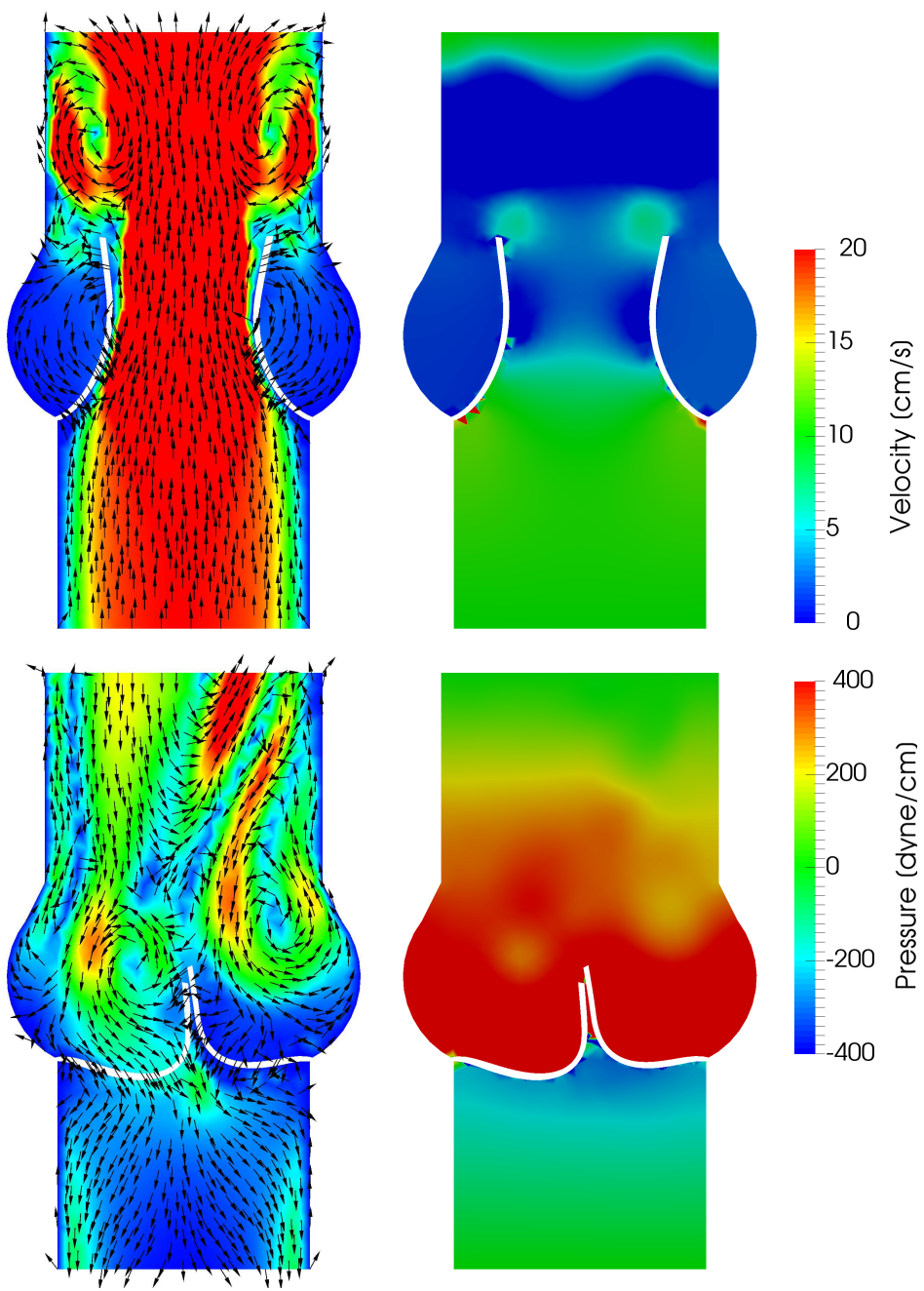

Figure 14: Plot of the velocity (left) and pressure (right) fields at time $t=0.375 \mathrm{~s}$ (top) and $t=0.675 \mathrm{~s}$ (bottom) (test II).

This test demonstrates that the proposed method can be successfully employed to simulate complex dynamics as in the case of cardiac valves, where the immersed structures undergo large displacements and may come into contact. Moreover, we notice that the proposed PolyDG method seems to be robust with respect to high Reynolds numbers.

\section{Conclusions}

We have proposed a new method that is able to simulate fluid-structure interaction problems in the case of immersed structures that exhibit large displacements and come into contact. In particular, we have introduced a consistent Discontinuous Galerkin method 
on polygonal/polyhedral grids that incorporates naturally the contact conditions in the formulation via the DG paradigm and that can handle naturally polygonal/polyhedral grids in any space dimension and for any approximation order. The results showed the effectiveness of the method to deal with the FSI contact problem also in complex configurations such as valve dynamics and for high-order discretizations.

We observe that we have employed a DG paradigm also far from the fluid-solid interface in order to be able in the future to locally tune the discretization parameters, namely the mesh size and the polynomial approximation degree, in an elementwise manner. This allows to save the total number of degrees of freedom and thus improve efficiency, which is mandatory in view of three-dimensional applications. Regarding the contact in a FSI framework, this could be a limit of the method compared to others, such as the CutFEM in [2] and the fully-Eulerian approach in [24], which allow the possibility to use the continuous Finite Element method far from the interfaces and thus reducing the computational cost.

Further research on the development and the analysis of algorithms for the selfcontact and the extension to the 3D case are under investigation. Another issue in the context of polygonal elements methods is the analysis of inf-sup stable discrete spaces for the velocity and pressure in the fluid saddle-point problem that guarantee the inf-sup stability under mild geometric requirements. Also this point is under investigation.

\section{Acknowledgments}

The authors are members of the INdAM Research group GNCS and this work is partially funded by INDAM-GNCS. PFA and SZ have been partially funded by the Italian research project MIUR PRIN17, number 201744KLJL. CV has been partially supported by the H2020-MSCA-ITN-2017, EU project 765374 "ROMSOC - Reduced Order Modelling, Simulation and Optimization of Coupled systems" and by the Italian research project MIUR PRIN17 2017AXL54F "Modeling the heart across the scales: from cardiac cells to the whole organ".

\section{References}

[1] C. Ager, B. Schott, A.-T. Vuong, A. Popp, and W. A. Wall. A consistent approach for fluidstructure-contact interaction based on a porous flow model for rough surface contact. International Journal for Numerical Methods in Engineering, 2019.

[2] C. Ager, A. Seitz, and W. A. Wall. A consistent and versatile computational approach for general fluid-structure-contact interaction problems. International Journal for Numerical Methods in Engineering, 2020.

[3] J. Aghili, S. Boyaval, and D. A. Di Pietro. Hybridization of Mixed High-Order Methods on General Meshes and Application to the Stokes Equations. Computational Methods in Applied Mathematics, 15(2):111-134, 2015. 
[4] F. Alauzet, B. Fabrèges, M. A. Fernández, and M. Landajuela. Nitsche-XFEM for the coupling of an incompressible fluid with immersed thin-walled structures. Computer Methods in Applied Mechanics and Engineering, 301:300-335, 2016.

[5] P. F. Antonietti, L. Beirão Da Veiga, D. Mora, and M. Verani. A Stream Virtual Element Formulation of the Stokes Problem on Polygonal Meshes. SIAM Journal on Numerical Analysis, 52(1):386-404, 2014.

[6] P. F. Antonietti, F. Brezzi, and L. D. Marini. Bubble stabilization of Discontinuous Galerkin methods. Computer Methods in Applied Mechanics and Engineering, 21-26(198):1651-1659, 2009.

[7] P. F. Antonietti, A. Dedner, P. Madhavan, S. Stangalino, B. Stinner, and M. Verani. High order discontinuous Galerkin methods for elliptic problems on surfaces. SIAM Journal on Numerical Analysis, 53(2):1145-1171, 2015.

[8] P. F. Antonietti, C. Facciola, P. Houston, I. Mazzieri, G. Pennesi, and M. Verani. High-Order Discontinuous Galerkin Methods on Polyhedral Grids for Geophysical Applications: Seismic Wave Propagation and Fractured Reservoir Simulations. In SEMA-SIMAI Springer series on Polyhedral Methods in Geosciences. Springer, 2020.

[9] P. F. Antonietti, P. Houston, and G. Pennesi. Fast Numerical Integration on Polytopic Meshes with Applications to Discontinuous Galerkin Finite Element Methods. Journal of Scientific Computing, 77(3):1339-1370, 2018.

[10] P. F. Antonietti and I. Mazzieri. High-order Discontinuous Galerkin methods for the elastodynamics problem on polygonal and polyhedral meshes. Computer Methods in Applied Mechanics and Engineering, 342:414-437, 2018.

[11] P. F. Antonietti, M. Verani, C. Vergara, and S. Zonca. Numerical solution of fluid-structure interaction problems by means of a high order Discontinuous Galerkin method on polygonal grids. Finite Elements in Analysis and Design, 159:1-14, 2019.

[12] M. Astorino, J.-F. Gerbeau, O. Pantz, and K.-F. Traoré. Fluid-structure interaction and multibody contact: Application to the aortic valves. Computer Methods in Applied Mechanics and Engineering, 198:3603-3612, 2009.

[13] N.J. Balmforth, C.J. Cawthorn, and R.V. Craster. Contact in a viscous fluid. Part 2. A compressible fluid and an elastic solid. Journal of Fluid Mechanics, 646:339-361, 2010.

[14] F. Bassi, L. Botti, A. Colombo, D. A. Di Pietro, and P. Tesini. On the flexibility of agglomeration based physical space discontinuous Galerkin discretizations. Journal of Computational Physics, 231(1):45-65, 2012.

[15] L. Beirão da Veiga, C. Canuto, R. H. Nochetto, and G. Vacca. Equilibrium analysis of an immersed rigid leaflet by the virtual element method, 2020.

[16] L. Beirão da Veiga, C. Lovadina, and G. Vacca. Virtual Elements for the Navier-Stokes Problem on Polygonal Meshes. SIAM Journal on Numerical Analysis, 56(3):1210-1242, 2018.

[17] L. Beirão da Veiga, D. Mora, and G. Vacca. The Stokes Complex for Virtual Elements with Application to Navier-Stokes Flows. Journal of Scientific Computing, 81(2):990-1018, 2019.

[18] L. Bocquet and J.-L. Barrat. Flow boundary conditions from nano- to micro-scales. Soft Matter, 3(6):685-693, 2007.

[19] D. Boffi and L. Gastaldi. Discrete models for fluid-structure interactions: the Finite Element Immersed Boundary Method. Discrete and Continuous Dynamical Systems, Series S(9):89-107, 2016.

[20] L. Boilevin-Kayl, M. A. Fernández, and J.-F. Gerbeau. A Loosely Coupled Scheme for Fictitious Domain Approximations of Fluid-Structure Interaction Problems with Immersed ThinWalled Structures. SIAM Journal on Scientific Computing, 41(2):B351-B374, 2019.

[21] I. Borazjani. Fluid-structure interaction, immersed boundary-finite element method simula- 
tions of bio-prosthetic heart valves. Computer Methods in Applied Mechanics and Engineering, 257:103-116, 2013.

[22] L. Botti, D. A. Di Pietro, and J. Droniou. A Hybrid High-Order method for the incompressible Navier-Stokes equations based on Temam's device. Journal of Computational Physics, 376:786-816, 2019.

[23] E. Burman and M. A. Fernández. An unfitted Nitsche method for incompressible fluidstructure interaction using overlapping meshes. Computer Methods in Applied Mechanics and Engineering, 279:497-514, 2014.

[24] E. Burman, M. A. Fernández, and S. Frei. A Nitsche-based formulation for fluid-structure interactions with contact. ESAIM: Mathematical Modelling and Numerical Analysis, 54(2):531$564,2020$.

[25] E. Burman, P. Hansbo, and M. G. Larson. Augmented Lagrangian and Galerkin least-squares methods for membrane contact. International Journal for Numerical Methods in Engineering, 114(11):1179-1191, 2018.

[26] E. Burman, P. Hansbo, and M. G. Larson. Augmented Lagrangian finite element methods for contact problems. ESAIM: Mathematical Modelling and Numerical Analysis, 53(1):173-195, 2019.

[27] E. Burman, P. Hansbo, M. G. Larson, and R. Stenberg. Galerkin least squares finite element method for the obstacle problem. Computer Methods in Applied Mechanics and Engineering, 313:362-374, 2017.

[28] A. Cangiani, Z. Dong, and E. H. Georgoulis. $h p$-Version Space-Time Discontinuous Galerkin Methods for Parabolic Problems on Prismatic Meshes. SIAM Journal on Scientific Computing, 39(4):A1251-A1279, 2017.

[29] A. Cangiani, Z. Dong, and E. H. Georgoulis. $h p$-Version discontinuous Galerkin methods on essentially arbitrarily-shaped elements. arXiv preprint arXiv:1906.01715, 2019.

[30] A. Cangiani, Z. Dong, E. H. Georgoulis, and P. Houston. hp-Version discontinuous Galerkin methods for advection-diffusion-reaction problems on polytopic meshes. ESAIM: Mathematical Modelling and Numerical Analysis, 50(3):699-725, 2016.

[31] A. Cangiani, Z. Dong, E. H. Georgoulis, and P. Houston. hp-Version Discontinuous Galerkin Methods on Polygonal and Polyhedral Meshes. Springer, 2017.

[32] A. Cangiani, E. H. Georgoulis, and P. Houston. $h p$-Version discontinuous Galerkin methods on polygonal and polyhedral meshes. Mathematical Models and Methods in Applied Sciences, 24(10):2009-2041, 2014.

[33] A. Cangiani, V. Gyrya, and G. Manzini. The NonConforming Virtual Element Method for the Stokes Equations. SIAM Journal on Numerical Analysis, 54(6):3411-3435, 2016.

[34] C.J. Cawthorn and N.J. Balmforth. Contact in a viscous fluid. Part 1. A falling wedge. Journal of Fluid Mechanics, 646:327-338, 2010.

[35] F. Chouly, A. Ern, and N. Pignet. A Hybrid High-Order discretization combined with Nitsche's method for contact and Tresca friction in small strain elasticity. SIAM Journal on Scientific Computing, 42(4):A2300-A2324, 2020.

[36] F. Chouly, M. Fabre, P. Hild, R. Mlika, J. Pousin, and Y. Renard. An Overview of Recent Results on Nitsche's Method for Contact Problems. In Geometrically Unfitted Finite Element Methods and Applications, pages 93-141. Springer, 2017.

[37] F. Chouly and P. Hild. A Nitsche-Based Method for Unilateral Contact Problems: Numerical Analysis. SIAM Journal on Numerical Analysis, 51(2):1295-1307, 2013.

[38] F. Chouly and P. Hild. On convergence of the penalty method for unilateral contact problems. Applied Numerical Mathematics, 65:27-40, 2013. 
[39] F. Chouly, R. Mlika, and Y. Renard. An unbiased Nitsche's approximation of the frictional contact between two elastic structures. Numerische Mathematik, 139(3):593-631, 2018.

[40] F. Chouly and Y. Renard. Explicit Verlet time-integration for a Nitsche-based approximation of elastodynamic contact problems. Advanced Modeling and Simulation in Engineering Sciences, 5(1):31, 2018.

[41] S. Claus and P. Kerfriden. A stable and optimally convergent LaTIn-CutFEM algorithm for multiple unilateral contact problems. International Journal for Numerical Methods in Engineering, 113(6):938-966, 2018.

[42] D. A. Di Pietro and A. Ern. Discrete functional analysis tools for Discontinuous Galerkin methods with application to the incompressible Navier-Stokes equations. Mathematics of Computation, 79(271):1303-1330, 2010.

[43] D. A. Di Pietro and S. Krell. A Hybrid High-Order Method for the Steady Incompressible Navier-Stokes Problem. Journal of Scientific Computing, 74(3):1677-1705, 2018.

[44] T. Dickopf and R. Krause. Efficient simulation of multi-body contact problems on complex geometries: A flexible decomposition approach using constrained minimization. International Journal for Numerical Methods in Engineering, 77(13):1834-1862, 2009.

[45] N. Diniz dos Santos, J.-F. Gerbeau, and J.-F. Bourgat. A partitioned fluid-structure algorithm for elastic thin valves with contact. Computer Methods in Applied Mechanics and Engineering, 197(19-20):1750-1761, 2008.

[46] J. Donea. An arbitrary Lagrangian-Eulerian finite element method for transient dynamic fluid-structure interaction. Computer Methods in Applied Mechanics and Engineering, 33:689$723,1982$.

[47] Q. Du, M. D. Gunzburger, L. S. Hou, and J. Lee. Semidiscrete finite element approximations of a linear fluid-structure interaction problem. SIAM Journal on Numerical Analysis, 42(1):129, 2004.

[48] C. Farhat, M. Lesoinne, and P. Le Tallec. Load and motion transfer algorithms for fluid/structure interaction problems with non-matching discrete interfaces: Momentum and energy conservation, optimal discretization and application to aeroelasticity. Computer Methods in Applied Mechanics and Engineering, 157(1-2):95-114, 1998.

[49] S. Frei. Eulerian finite element methods for interface problems and fluid-structure interactions. PhD thesis, Heidelberg University, 2016.

[50] D. Gérard-Varet and M. Hillairet. Regularity Issues in the Problem of Fluid Structure Interaction. Archive for Rational Mechanics and Analysis, 195(2):375-407, 2010.

[51] D. Gérard-Varet, M. Hillairet, and C. Wang. The influence of boundary conditions on the contact problem in a 3D Navier-Stokes flow. Journal de Mathématiques Pures et Appliquées, 103(1):1-38, 2015.

[52] J.-F. Gerbeau and M. Vidrascu. A quasi-Newton algorithm based on a reduced model for fluid-structure interaction problems in blood flows. ESAIM: Mathematical Modelling and $\mathrm{Nu}$ merical Analysis, 37(4):631-647, 2003.

[53] A. Gerstenberger and W. A. Wall. An extended finite element method/Lagrange multiplier based approach for fluid-structure interaction. Computer Methods in Applied Mechanics and Engineering, 197(19):1699-1714, 2008.

[54] B. E. Griffith. Immersed boundary model of aortic heart valve dynamics with physiological driving and loading conditions. International Journal for Numerical Methods in Biomedical Engineering, 28(3):317-345, 2012.

[55] E. Hairer, S. P. Nørsett, and G. Wanner. Solving Ordinary Differential Equations I. Nonstiff Problems. Springer-Verlag Berlin Heidelberg, 1993. 
[56] R. Haj-Ali, G. Marom, S. B. Zekry, M. Rosenfeld, and E. Raanani. A general threedimensional parametric geometry of the native aortic valve and root for biomechanical modeling. Journal of biomechanics, 45(14):2392-2397, 2012.

[57] M. Hillairet and T. Takahashi. Collisions in Three-Dimensional Fluid Structure Interaction Problems. SIAM Journal on Mathematical Analysis, 40(6):2451-2477, 2009.

[58] S. Hüeber, G. Stadler, and B. Wohlmuth. A Primal-Dual Active Set Algorithm for ThreeDimensional Contact Problems with Coulomb Friction. SIAM Journal on Scientific Computing, 30(2):572-596, 2008.

[59] S. Hüeber and B. Wohlmuth. A primal-dual active set strategy for non-linear multibody contact problems. Computer Methods in Applied Mechanics and Engineering, 194(27-29):31473166, 2005.

[60] N. Kikuchi and J. T. Oden. Contact Problems in Elasticity: A Study of Variational Inequalities and Finite Element Methods, volume 8. siam, 1988.

[61] R. Krause and B. Wohlmuth. A Dirichlet-Neumann type algorithm for contact problems with friction. Computing and Visualization in Science, 5(3):139-148, 2002.

[62] E. Lauga, M. Brenner, and H. Stone. Microfluidics: The No-Slip Boundary Condition. Springer Handbook of Experimental Fluid Mechanics, pages 1219-1240, 2007.

[63] C. Lehrenfeld. High order unfitted finite element methods on level set domains using isoparametric mappings. Computer Methods in Applied Mechanics and Engineering, 300:716733, 2016.

[64] K. Lipnikov, D. Vassilev, and I. Yotov. Discontinuous Galerkin and mimetic finite difference methods for coupled Stokes-Darcy flows on polygonal and polyhedral grids. Numerische Mathematik, 126(2):321-360, 2014.

[65] G. Marom. Numerical Methods for Fluid-Structure Interaction Models of Aortic Valves. Archives of Computational Methods in Engineering, 22(4):595-620, 2015.

[66] J.E. Marsden and T.J.R. Hughes. Mathematical Foundations of Elasticity. Dover Civil and Mechanical Engineering. Dover, 1984.

[67] A. Massing, M. G. Larson, A. Logg, and M. E. Rognes. A Nitsche-based cut finite element method for a fluid-structure interaction problem. Communications in Applied Mathematics and Computational Science, 10(2):97-120, 2015.

[68] U. M. Mayer, A. Popp, A. Gerstenberger, and W. A. Wall. 3D fluid-structure-contact interaction based on a combined XFEM FSI and dual mortar contact approach. Computational Mechanics, 46(1):53-67, 2010.

[69] R. Mlika, Y. Renard, and F. Chouly. An unbiased Nitsche's formulation of large deformation frictional contact and self-contact. Computer Methods in Applied Mechanics and Engineering, 325:265-288, 2017.

[70] M. E. Moghadam, Y. Bazilevs, T.-Y. Hsia, I. E Vignon-Clementel, A. L Marsden, and Modeling of Congenital Hearts Alliance (MOCHA). A comparison of outlet boundary treatments for prevention of backflow divergence with relevance to blood flow simulations. Computational Mechanics, 48(3):277-291, 2011.

[71] C. Neto, D. R. Evans, E. Bonaccurso, H.-J. Butt, and V. S. J. Craig. Boundary slip in Newtonian liquids: a review of experimental studies. Reports on Progress in Physics, 68(12):2859, 2005.

[72] F. Nobile and C. Vergara. An effective fluid-structure interaction formulation for vascular dynamics by generalized Robin conditions. SIAM Journal on Scientific Computing, 30(2):731763, 2008.

[73] W. Pazner and P. O. Persson. On the convergence of iterative solvers for polygonal discon- 
tinuous Galerkin discretizations. Communications in Applied Mathematics and Computational Science, 13:27-51, 2018.

[74] C. S. Peskin. Flow patterns around heart valves: A numerical method. Journal of Computational Physics, 10(2):252-271, 1972.

[75] A. Popp, A. Seitz, M. W. Gee, and W. A. Wall. Improved robustness and consistency of 3D contact algorithms based on a dual mortar approach. Computer Methods in Applied Mechanics and Engineering, 264:67-80, 2013.

[76] M. A. Puso and T. A. Laursen. A mortar segment-to-segment frictional contact method for large deformations. Computer Methods in Applied Mechanics and Engineering, 193(45-47):48914913, 2004.

[77] T. Richter. A Fully Eulerian formulation for fluid-structure-interaction problems. Journal of Computational Physics, 233:227-240, 2013.

[78] S. Sathe and T. E. Tezduyar. Modeling of fluid-structure interactions with the space-time finite elements: contact problems. Computational Mechanics, 43(1):51, 2008.

[79] T. E. Tezduyar and S. Sathe. Modelling of fluid-structure interactions with the space-time finite elements: Solution techniques. International Journal for Numerical Methods in Fluids, 54(6-8):855-900, 2007.

[80] R. van Loon, P. D. Anderson, and F. N. van de Vosse. A fluid-structure interaction method with solid-rigid contact for heart valve dynamics. Journal of Computational Physics, 217(2):806-823, 2006.

[81] E. Votta, T. B. Le, M. Stevanella, L. Fusini, E. G. Caiani, A. Redaelli, and F. Sotiropoulos. Toward patient-specific simulations of cardiac valves: State-of-the-art and future directions. Journal of Biomechanics, 46(2):217-228, 2013.

[82] D. Wiresaet, E. J. Kubatko, C. E. Michoski, S. Tanaka, J. J. Westerink, and C. Dawson. Discontinuous Galerkin methods with nodal and hybrid modal/nodal triangular, quadrilateral, and polygonal elements for nonlinear shallow water flow. Computer Methods in Applied Mechanics and Engineering, 270:113-149, 2014.

[83] B. Wohlmuth. Variationally consistent discretization schemes and numerical algorithms for contact problems. Acta Numerica, 20:569-734, 2011.

[84] B. Wohlmuth and R. Krause. Monotone Multigrid Methods on Nonmatching Grids for Nonlinear Multibody Contact Problems. SIAM Journal on Scientific Computing, 25(1):324-347, 2003.

[85] P. Wriggers and G. Zavarise. Computational Contact Mechanics. Encyclopedia of Computational Mechanics, 2004.

[86] Z. Yu. A DLM/FD method for fluid/flexible-body interactions. Journal of Computational Physics, 207(1):1-27, 2005.

[87] L. Zhang, M. Cai, and M. Mu. A multirate approach for fluid-structure interaction computation with decoupled methods. Communications in Computational Physics, 27(4):1014-1031, 2020.

[88] S. Zonca, C. Vergara, and L. Formaggia. An unfitted formulation for the interaction of an incompressible fluid with a thick structure via an XFEM/DG approach. SIAM Journal on Scientific Computing, 40(1):B59-B84, 2018. 